PAPER

\section{Electron cyclotron heating can drastically alter reversed shear Alfvén eigenmode activity in DIII-D through finite pressure effects}

To cite this article: M.A. Van Zeeland et al 2016 Nucl. Fusion 56112007

View the article online for updates and enhancements.

\section{Related content}

- Measurements, modelling and ECH
$\frac{\text { modification of AE activity in DIII-D }}{\text { M.A. Van Zeeland, W.W. Heidbrink, R. }}$
Nazikian et al.
- Energetic particle physics in fusion
$\frac{\text { research in preparation for burning plasma }}{\text { experiments }}$
N.N. Gorelenkov, S.D. Pinches and K. Toi
- Phase-space dependent critical gradient
behavior of fast-ion transport due to Alfvén
eigenmodes
C.S. Collins, W.W. Heidbrink, M. Podestà
et al.

\section{Recent citations}

- KSTAR Status and Upgrade Plan Toward
$\frac{\text { Fusion Reactor }}{\text { Jong-Gu Kwak et al }}$
- $\frac{\text { Simulation of the eigenmode spectrum }}{\text { below the Toroidicity-induced Alfvén }}$
$\frac{\text { eigenmode gap generated by the coupling }}{\text { of Alfvén and slow-magnetosonic waves in }}$
$\frac{\text { tokamaks }}{\text { G J Kramer et al }}$
- Exploring fusion-reactor physics with high-
power electron cyclotron resonance
heating on ASDEX Upgrade
J Stober et al




\title{
Electron cyclotron heating can drastically alter reversed shear Alfvén eigenmode activity in DIII-D through finite pressure effects
}

\author{
M.A. Van Zeeland ${ }^{1}$, W.W. Heidbrink ${ }^{2}$, S.E. Sharapov ${ }^{3}$, D. Spong ${ }^{4}$, A. Cappa ${ }^{5}$, \\ Xi Chen ${ }^{1}$, C. Collins ${ }^{2}$, M. García-Muñoz ${ }^{6}$, N.N. Gorelenkov ${ }^{7}$, G.J. Kramer ${ }^{7}$, \\ P. Lauber ${ }^{6}$ Z. Lin ${ }^{2}$ and C. Petty ${ }^{1}$ \\ 1 General Atomics, PO Box 85608 San Diego, CA 92186-5608, USA \\ 2 University of California at Irvine, Irvine, CA 92697, USA \\ ${ }^{3}$ CCFE, Culham Science Centre, Abingdon, Oxon, OX14 3DB, UK \\ ${ }^{4}$ Oak Ridge National Laboratory, Oak Ridge, TN 37830, USA \\ ${ }^{5}$ Laboratorio Nacional de Fusion-CIEMAT. 28040 Madrid, Spain \\ ${ }^{6}$ Max-Planck-Institut für Plasmaphysik, Euratom Association, Garching, Germany \\ 7 Princeton Plasma Physics Laboratory, PO Box 451, Princeton, NJ 08543-0451, USA \\ E-mail: vanzeeland@fusion.gat.com
}

Received 19 January 2016, revised 22 March 2016

Accepted for publication 7 April 2016

Published 22 July 2016

\begin{abstract}
A recent DIII-D experiment investigating the impact of electron cyclotron heating (ECH) on neutral beam driven reversed shear Alfvén eigenmode (RSAE) activity is presented. The experiment includes variations of ECH injection location and timing, current ramp rate, beam injection geometry (on/off-axis), and neutral beam power. Essentially all variations carried out in this experiment were observed to change the impact of ECH on AE activity significantly. In some cases, RSAEs were observed to be enhanced with ECH near the off-axis minimum in magnetic safety factor $\left(q_{\mathrm{min}}\right)$, in contrast to the original DIII-D experiments where the modes were absent when ECH was deposited near $q_{\text {min }}$. It is found that during intervals when the geodesic acoustic mode (GAM) frequency at $q_{\min }$ is elevated and the calculated RSAE minimum frequency, including contributions from thermal plasma gradients, is very near or above the nominal TAE frequency $\left(f_{\mathrm{TAE}}\right)$, RSAE activity is not observed or RSAEs with a much reduced frequency sweep range are found. This condition is primarily brought about by ECH modification of the local electron temperature $\left(T_{\mathrm{e}}\right)$ which can raise both the local $T_{\mathrm{e}}$ at $q_{\min }$ as well as its gradient. A $q$-evolution model that incorporates this reduction in RSAE frequency sweep range is in agreement with the observed spectra and appears to capture the relative balance of TAE or RSAE-like modes throughout the current ramp phase of over 38 DIII-D discharges. Detailed ideal MHD calculations using the NOVA code show both modification of plasma pressure and pressure gradient at $q_{\text {min }}$ play an important role in modifying the RSAE activity. Analysis of the ECH injection near the $q_{\text {min }}$ case where no frequency sweeping RSAEs are observed shows the typical RSAE is no longer an eigenmode of the system. What remains is an eigenmode with poloidal harmonic content reminiscent of the standard RSAE, but absent of the typical frequency sweeping behavior. The remaining eigenmode is also often strongly coupled to gap TAEs. Analysis with the non-perturbative gyro fluid code TAEFL confirms this change in RSAE activity and also shows a large drop in the resultant mode growth rates.
\end{abstract}


Keywords: magnetohydrodynamic waves, tokamaks, fusion products effects, plasma heating by microwaves, plasma heating by particle beams

(Some figures may appear in colour only in the online journal)

\section{Introduction}

Localized electron cyclotron heating $(\mathrm{ECH})$ can have a dramatic effect on neutral beam driven Alfvén eigenmode (AE) activity as was first demonstrated in DIII-D reversed magnetic shear plasmas $[1,2]$ and later on several other devices worldwide including Heliotron-J [3], TJ-II [4], LHD [5] and ASDEX-Upgrade [6]. The most commonly observed effect on DIII-D is a shift in the dominant observed modes from a mix of reversed shear Alfvén eigenmodes (RSAEs) [7] and toroidicity induced Alfvén eigenmodes [8-10] (TAEs) to a spectrum of weaker TAEs when ECH is deposited near the shear reversal point $\left(q_{\min }\right)[1,2]$. ECH deposition near the magnetic axis typically increases the unstable mode amplitudes and resultant fast ion transport. Due to the fact that localized electron heating can impact essentially all aspects of AEs, including mode drive, damping, and the ideal eigenmode itself, a satisfactory explanation for this effect in DIII-D has been elusive. Mode stability is altered through modification of the different damping channels (electron collisional [12] and electron Landau [13] in particular, and even continuum damping [14] through pressure or rotation [15, 16] induced changes to the modes and continuum), mode drive ( $T_{\mathrm{e}}$ directly impacts electron drag on fast ions), and the AEs themselves through coupling to sound waves via changes to $T_{\mathrm{e}} / T_{\mathrm{i}}$ [17]. Recent predictions also indicate AE saturation and their impact on the fast ion profile can be affected by microturbulence [11] which can be modified by ECH. It is the point of this paper to determine the dominant physical mechanisms responsible for the change in RSAE activity in DIII-D plasmas when ECH is applied.

The RSAE has been investigated in detail since its original discovery [7] and is described in many references [19-26] but a brief review is given since several fundamental points are key to understanding the results presented here. The RSAE is basically a cylindrical mode with toroidal mode number $(n)$ and one dominant poloidal harmonic $(m)$. The RSAE appears when the minimum safety factor $\left(q_{\min }\right)$ is approximately $q_{\min } \approx m / n$ and for a decreasing $q_{\min }$, a second poloidal harmonic $m-1$ increases in amplitude until $q_{\min }=(m-1 / 2) / n$, at which point the RSAE becomes a toroidicity induced Alfvén eigenmode. Throughout this transition, the mode's frequency increases to the local TAE frequency $\left(f_{\mathrm{TAE}}\right)$. Of particular importance to the work presented here is the fact that the RSAE can be significantly modified by finite pressure effects and geodesic curvature which precludes the shear Alfvén wave continuum from going to zero frequency at a rational surface. Instead, the continuum is bounded by that of the geodesic acoustic mode [18] $\left(f_{\mathrm{GAM}}\right)$ and the RSAE minimum frequency $\left(f_{\mathrm{RSAE}-\mathrm{min}}\right)$ lies above the local continuum tip at $q_{\min }$ by an amount that depends on the local pressure as well as gradients in the local thermal and fast ion pressures [23-31]. ECH directly modifies the local electron temperature and temperature gradient and, in this way, can easily modify RSAEs as well.

A recent DIII-D experiment to understand the physical mechanisms responsible for the observed shift in RSAE activity with ECH utilized a simplified oval geometry and, in addition to $\mathrm{ECH}$ injection location, included variations of current ramp rate, $\mathrm{ECH}$ injection timing, beam injection geometry (on/off-axis), and neutral beam power. Essentially all variations carried out in this experiment were observed to change the impact of $\mathrm{ECH}$ on AE activity significantly. In this paper, we first discuss the basic discharge used then make connection with historical results showing a 'stabilization' of RSAE activity with ECH injection near mid-radius during the current ramp portion of the discharge (section 2). We then introduce a simple model for the RSAE spectra used to interpret the data from a large collection of discharges (section 3). Several experimental cases are then examined and it is shown that the previously observed impact of ECH on RSAE activity is not ubiquitous and, in fact, the opposite effect can be observed, e.g. RSAEs can be enhanced by application of ECH at $q_{\min }$ by simply delaying the injection timing (section 4 ). It is then shown that the ratio of the predicted RSAE minimum frequency to the TAE frequency is the dominant factor determining the presence of typical frequency sweeping RSAEs, similar to the so-called beta suppression mechanism put forward to explain the absence of typical frequency sweeping RSAEs in spherical tokamaks [32]. In the last section (section 5), analyses from the ideal MHD code NOVA [42, 43] and non-perturbative gyrofluid code TAEFL [44] are presented where it is shown that both modification of plasma pressure and pressure gradient at $q_{\text {min }}$ play an important role in modifying the RSAE activity. ECH can modify the pressure profile such that the typical frequency sweeping RSAE is no longer an eigenmode of the system and a large drop in the resultant mode growth rates is found.

\section{Discharge background and reproduction of historial ech/ae results in oval plasmas}

The majority of discharges presented in this paper utilize an oval (elongation, $\kappa \approx 1.5$ ) shaped L-mode deuterium plasma with either no ECH or radial launch, 2nd harmonic, $110 \mathrm{GHz}$ electron cyclotron heating directed to two possible locations, one near the magnetic axis and the other near mid-radius (chosen to be near $q_{\text {min }}$ during the current ramp portion of the discharge). A representative EFIT [33] equilibrium reconstruction is shown in figure 1 , along with the two primary ECH steering trajectories. In these $B_{\mathrm{T}}=2.05 \mathrm{~T}$ discharges, $75-80 \mathrm{kV}$ sub-Alfvénic neutral beam injection begins 


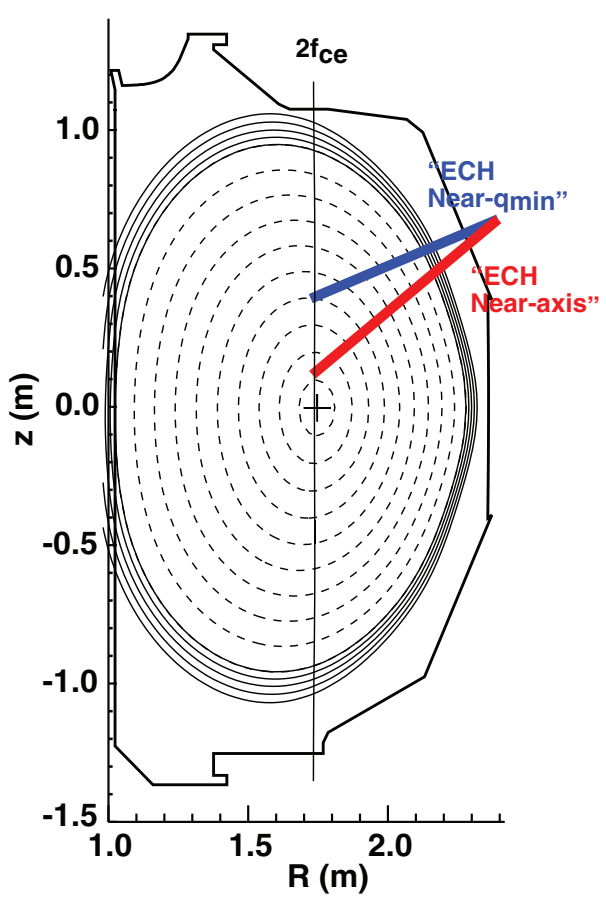

Figure 1. Representative equilibrium reconstruction with ECH steering trajectories overlaid. Dashed lines indicate equally spaced contours of normalized toroidal flux from 0.1-1.0. Actual EFIT is for discharge $157736, t=431 \mathrm{~ms}$.

at $t=300 \mathrm{~ms}$ and continues while the plasma current $\left(I_{p}\right)$ is ramping up at a constant rate of $0.8 \mathrm{MA} \mathrm{s}^{-1}$ until reaching approximately $1.2 \mathrm{MA}$ at $t=1100 \mathrm{~ms}$. Time histories of plasma current, $q_{\min }$, line-averaged electron density $\left(n_{\mathrm{e}}\right)$, electron temperature at $q_{\min }$, injected neutral beam $\left(P_{\mathrm{NBI}}\right)$ and electron cyclotron heating power $\left(P_{\mathrm{ECH}}\right)$ are shown in figure 2 for two discharges that will be discussed in detail-157735 (ECH near $q_{\text {min }}$ ) and 157737 (No ECH). In these discharges electron temperature is diagnosed by a 40 channel electron cyclotron emission (ECE) diagnostic [34] as well as Thomson Scattering (which also measures electron density). Ion temperature and rotation are inferred through measurements of carbon impurities using charge exchange recombination spectroscopy (CER). The safety factor profile is obtained using EFIT reconstructions constrained by a motional Stark effect (MSE) polarimetry system. From the time histories, it can be seen that the addition of $\mathrm{ECH}$, as expected, causes a significant increase in electron temperature. The difference in $T_{\mathrm{e}}$, however, eventually diminishes and will be discussed, along with the implications, in more detail in section 4 . The added electron heating also causes significant differences in the safety factor profile as can be seen in figure 3 , where radial profiles of $q, T_{\mathrm{e}}, T_{\mathrm{i}}, n_{\mathrm{e}}$, toroidal rotation $\left(f_{\text {rot }}\right)$, equilibrium pressure, and NBI pressure at $q_{\min }=4$ in discharges 157735 and 157737 are given. These profiles will be used as inputs for TAEFL and NOVA modeling, to be discussed later in section 5 . The primary differences in these two cases are in the $q$-profile (figure 3(a)) and electron temperature profile (figure 3(b)). For ECH near $q_{\min }$, the central safety factor is elevated relative to the no $\mathrm{ECH}$ case. This is understandable since we are looking during the current ramp portion of the discharge and the higher central
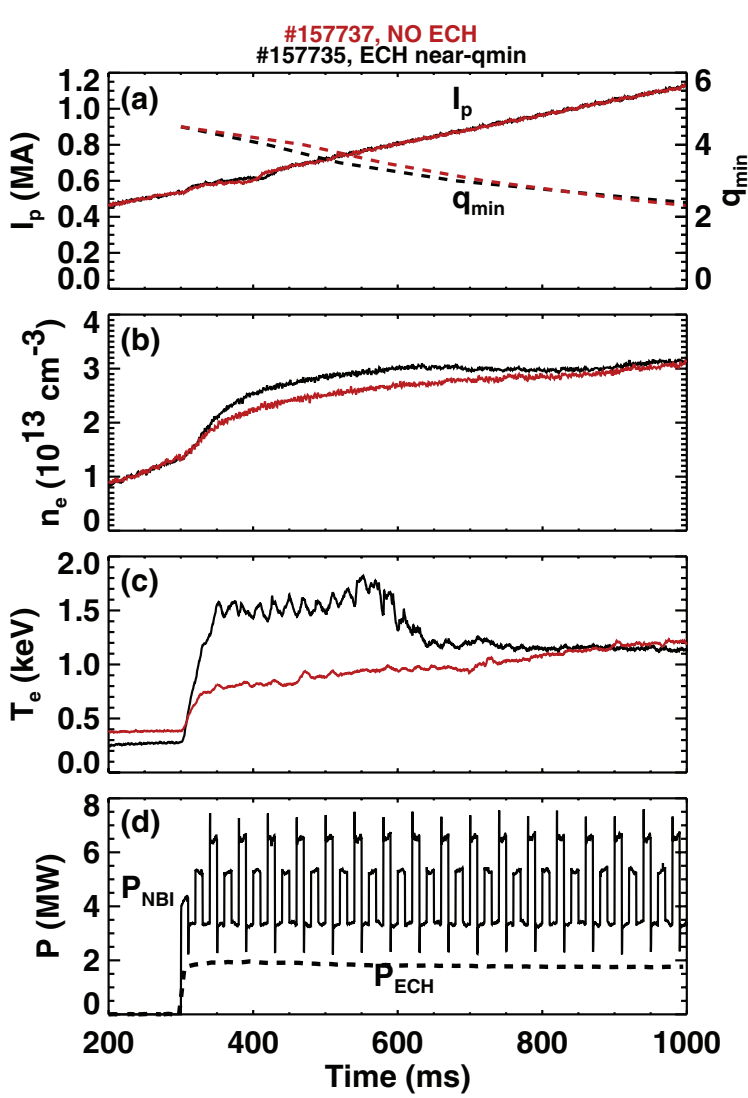

Figure 2. Data from DIII-D discharges 157735 (black) and 157737 (red). Time histories of $(a)$ plasma current $\left(I_{p}\right)$ and $q_{\min },(b)$ lineaveraged electron density $\left(n_{\mathrm{e}}\right),(c)$ electron temperature at $q_{\min }$ and, $(d)$ injected neutral beam $\left(P_{\mathrm{NBI}}\right)$ and electron cyclotron heating power $\left(P_{\mathrm{ECH}}\right)$. Note, 157735 has ECH near $q_{\text {min }}, 157737$ has no ECH.

electron temperature, due to heating, causes a delay in the current penetration to the axis. Interestingly, the ion temperature profiles are very similar in both cases.

Early neutral beam injection during the current ramp phase produces a variety of Alfvénic activity in DIII-D plasmas including toroidicity induced Alfvén eigenmodes, reversed shear Alfvén eigenmodes, and linearly coupled RSAEs and TAEs $[2,45,46]$. Spectrograms showing the rich variety of modes that are possible as well as the change in mode activity as ECH is directed to the two different steering locations are presented in figure 4. In this figure, as well several others throughout this paper, a composite spectrogram is constructed by averaging the cross power spectrum of several adjacent ECE channels. Discharge 157737 in figure 4(a) is a reproduction of a very well documented beam driven AE discharge in DIII-D (142111) that has been studied and modeled extensively with great success [35-41]. It was the intent of this experiment to begin with this well understood discharge and then move away from it using ECH. Discharges 157736 and 157735 have ECH injection beginning at $t=300 \mathrm{~ms}$ near the magnetic axis and $q_{\min }$ respectively. Consistent with historical results carried out in Dee shaped plasmas $[1,2]$ these new oval discharges show a radical shift in AE activity from a multitude of TAEs and RSAEs with ECH near the magnetic axis to a discharge that is strongly TAE dominated with very little, if any, observed frequency sweeping RSAEs with ECH near $q_{\text {min }}$. 


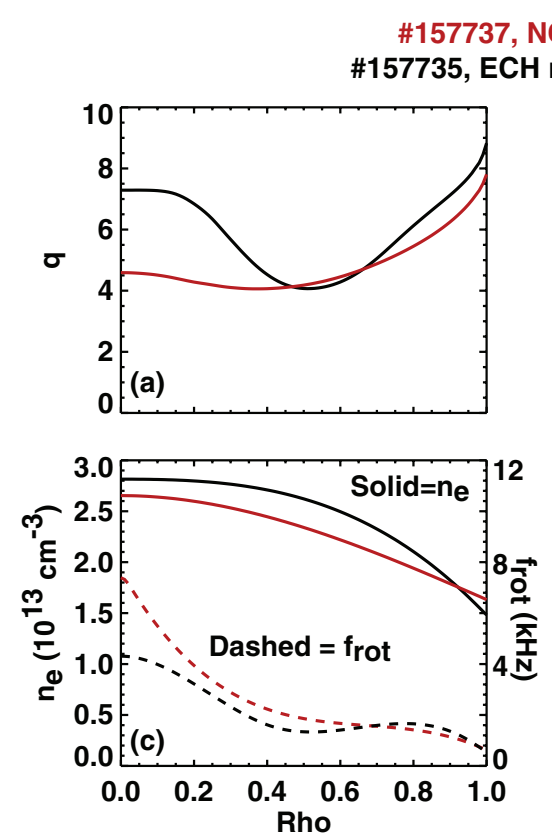

57737, NO ECH

57735, ECH near-qmin
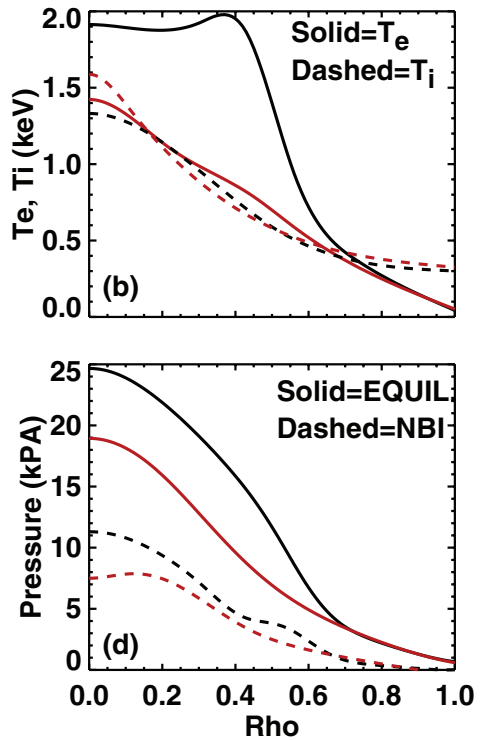

Figure 3. Radial profiles of $q, T_{\mathrm{e}}, T_{\mathrm{i}}, n_{\mathrm{e}}, f_{\text {rot }}$ equilibrium, and NBI pressure at $q_{\min }=4$ in $157735(t=430 \mathrm{~ms})$ with ECH near $q_{\min }$ and $157737(t=470 \mathrm{~ms})$ with NO ECH. Rho $=\rho=$ normalized square root of toroidal flux. Profiles used as inputs in NOVA and TAEFL modeling. (a) Safety factor profile, $(b)$ electron $\left(T_{\mathrm{e}}\right)$ and ion $\left(T_{\mathrm{i}}\right)$ temperature profiles, $(c)$ electron density $\left(n_{\mathrm{e}}\right)$, toroidal rotation $\left(f_{\text {rot }}\right)$, and $(d)$ equilibrium (solid) and beam ion (dashed) pressure profiles. NBI pressure is derived by subtracting thermal pressure from MSE constrained EFIT equilibrium pressure.

\section{Model for RSAE frequency evolution}

To aid in the interpretation of these results, a model for the RSAE spectral evolution throughout the current ramp is employed. Similar models have been described in the past and are frequently used to identify the temporal evolution of $q_{\min }$ through so-called AE or MHD spectroscopy [21, 47-50]. The model here is based on constraining the RSAE frequency sweep to be between some minimum frequency $\left(f_{\text {RSAE-min }}\right)$ and the TAE frequency $\left(f_{\mathrm{TAE}}\right)$. The RSAE minimum frequency lies above that of the geodesic acoustic mode $\left(f_{\mathrm{GAM}}\right)$ and below the TAE frequency $\left(f_{\mathrm{TAE}}\right)$. The reduction in RSAE frequency sweep range is a well known effect, as described in the introduction, and here, the RSAE minimum frequency is shifted above the local GAM frequency by an amount that depends on gradients of the thermal and fast ion pressures, the latter of which will be neglected, also other kinetic effects such as diamagnetic corrections or trapped particle effects, as described by more complete models in [51-53] are neglected here for simplicity. It is pointed out this model is used simply to survey a large dataset and capture some of the most important features and aspects affecting the ECH impact on RSAEs, which it does well. Such a survey cannot require detailed profile fitting, transport calculations, kinetic equilibrium reconstructions, or other analysis that would be unwieldy for such a large number of discharges and time ranges. It is not the authors' intention to present this as an all inclusive model for the RSAE spectral evolution, indeed, the impact of for example, the fast ion population on the RSAE frequency evolution is an ongoing topic of research [54]. More comprehensive calculations are introduced in the following section where ideal MHD NOVA calculations are presented, followed by non-perturbative
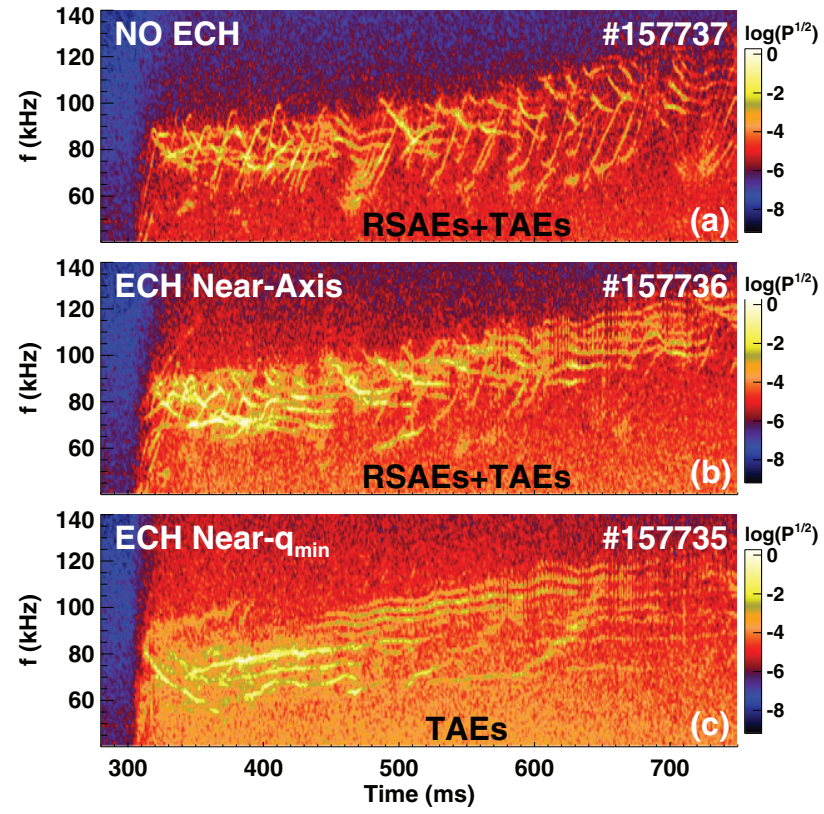

Figure 4. Combined ECE power spectra for discharges (a) 157737 with NO ECH, $(b) 157736$ with $\approx 1.1 \mathrm{MW} \mathrm{ECH}$ near the magnetic axis, and $(c) 157735$ with $\approx 1.9 \mathrm{MW}$ ECH near $q_{\text {min }}$.

TAEFL calculations which include the fast ions as a separate species. In this model, the relevant TAE and RSAE frequencies are given by:

$$
f_{\mathrm{TAE}}=\frac{V_{\mathrm{A}}}{4 \pi q_{\min } R}\left|\left(2-\left(1+3 \epsilon_{g}^{2} / 4\right)^{1 / 2}\right)\right| /\left(1-\epsilon_{g}^{2} / 4\right)^{1 / 2}
$$

where 

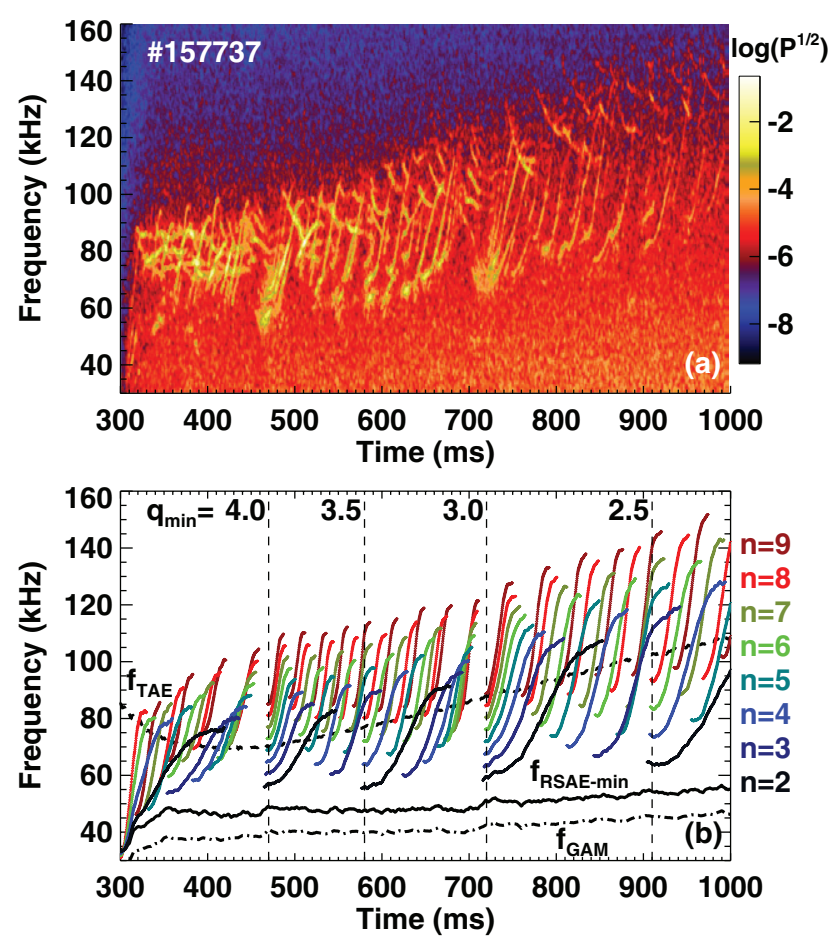

Figure 5. (a) Combined ECE spectrogram for discharge 157737 with No ECH heating. (b) Ad hoc model for RSAE frequency evolution. Dash-dot line indicates $f_{\mathrm{GAM}}$, dashed line indicates $f_{\mathrm{TAE}}$, and solid line indicates minimum RSAE frequency $f_{\text {RSAE-min }}$.

$$
\epsilon_{g}=\left(\kappa^{2}-1\right) /\left(\kappa^{2}+1\right)
$$

and

$$
f_{\mathrm{RSAE}-\min }^{2}=\left(f_{\mathrm{GAM}}^{2}+f_{\nabla}^{2}\right)
$$

with the GAM frequency given by

$f_{\mathrm{GAM}}^{2}=\frac{1}{4 \pi^{2}}\left[\frac{2}{M_{\mathrm{i}} R^{2}}\left(T_{\mathrm{e}}+\frac{7}{4} T_{\mathrm{i}}\right)\left(1+\frac{1}{2 q_{\text {min }}^{2}}\right)\right] \frac{2}{\kappa^{2}+1}$

and the upshift in RSAE minimum frequency from the GAM frequency due to thermal plasma temperature gradients

$$
f_{\nabla}^{2}=\frac{-1}{2 \pi^{2} M_{\mathrm{i}} R^{2}} \rho \frac{d}{d \rho}\left(T_{\mathrm{e}}+T_{\mathrm{i}}\right)\left(1-\frac{1}{q_{\min }^{2}}\right) \frac{2}{\kappa^{2}+1} .
$$

In the above, $\kappa$ is the plasma elongation, $V_{\mathrm{A}}$ is the Alfvén speed, $M_{\mathrm{i}}$ is the ion mass, $T_{\mathrm{i}}$ ion temperature, $T_{\mathrm{e}}$ electron temperature, and $R$ is major radius. The expressions for the TAE and GAM frequencies are modified from the standard cylindrical plasma expressions to include the effects of elongation and are adapted from [31] (also discussed in [55]) using $\gamma=1+3 / 4 \frac{T_{\mathrm{i}}}{T_{\mathrm{e}}+T_{\mathrm{i}}}$ for the ratio of specific heats. Without the elongation correction, the GAM frequency at high $q_{\text {min }}$ reduces to that in [24, 32]. The gradient induced frequency upshift $\left(f_{\nabla}\right)$ in $f_{\text {RSAE-min }}$ is from $[1,26$, 27] with the additional correction for elongation to be the same as that for $f_{\mathrm{GAM}}$ (see equation (11) in [31]). Note, we are ignoring contributions from the density gradient due to the fact that they are relatively small in the L-mode discharges presented here.
The actual ad hoc functional form for the frequency sweep of an individual RSAE, with poloidal mode number $m$ and toroidal mode number $n$, from $f_{\text {RSAE-min }}$ to $f_{\mathrm{TAE}}$ is chosen to give qualitatively similar behavior to experiment and the theoretical expectation that the minimum occurs at $q_{\min }=\mathrm{m} / \mathrm{n}$ and approaches $f_{\mathrm{TAE}}$ at $q_{\min } \approx(m-1 / 2) / n$. Here, we use:

$$
f_{\mathrm{RSAE}}=f_{\mathrm{RSAE}-\min }+f_{s} \frac{f_{\mathrm{TAE}}-f_{\mathrm{RSAE}-\mathrm{min}}}{\sqrt{2}-1}+n f_{\mathrm{rot}}
$$

where

$$
f_{s}=\left[\left(k_{0} /\left(k_{0}^{p}+1\right)^{1 / p}\right)^{2}+1\right]^{1 / 2}-1
$$

and

$$
k_{0}=2\left(m-n q_{\min }\right)
$$

with $p=9$ and $f_{\text {rot }}$ the toroidal rotation at $q_{\min }$. As will be shown, the relevant parameter for the impact of ECH on Alfvén eigenmodes discussed here is $f_{\text {RSAE-min }}$ relative to $f_{\text {TAE }}$. An example output of the model is shown in figure 5(b), where the measured spectrogram is given in figure 5(a). An important point is, for these and subsequent plots, $f_{\mathrm{RSAE}}$ is restricted to be less than $f_{\mathrm{TAE}}$ (excluding rotation). If $f_{\mathrm{RSAE}}$ goes above $f_{\mathrm{TAE}}$, it is clamped at $f_{\mathrm{TAE}}$ until it returns below. Also, in figure $5(b)$ and all future plots, each individual mode ( $m$ and $n$ ) is plotted only in the range $(m+0.03) / n<q_{\min }<(m-0.7) / n$. In each plot like that in figure $5(b)$, the local toroidal rotation at $q_{\min }$ appears as the Doppler shift induced separation between successive toroidal mode numbers. As can be seen from figure 5 there is excellent qualitative agreement between the model RSAE spectra and that measured. It is pointed out that not all modes plotted for a given $n$ have to be observed experimentally, that is a question of stability which is in no-way taken into account.

To give the reader an idea of how equations (1) and (3) for $f_{\mathrm{TAE}}$ and $f_{\mathrm{RSAE}-\mathrm{min}}$ agree with experiment, a comparison is made in figure 6. For this comparison, a database of 38 discharges was created in which spectra of ECE channels near $q_{\text {min }}$ were plotted and the minimum observed RSAE frequency (if any observable RSAE activity existed) as well as the lowest frequency TAE were tracked throughout the current ramp. The discharges examined have a large range of neutral beam and $\mathrm{ECH}$ heating scenarios, with neutral beam powers in the range $P_{\mathrm{NBI}} \approx 1-9 \mathrm{MW}$ and $P_{\mathrm{ECH}} \approx 0-3.4 \mathrm{MW}$. Figures $6(a)$ and $(b)$ are histograms of these measurements compared to the predictions for $f_{\mathrm{RSAE}-\min }$ and $f_{\mathrm{TAE}}$ at each condition. A separate point of comparison was created for every $5 \mathrm{~ms}$ throughout the current ramp of each of the 38 discharges. The comparison shows good agreement for both frequencies over the range of conditions examined. For the comparison, the measured frequencies are actually the measured minimum frequencies minus the local rotation frequency measured with CER at mid-radius. It is noted, this approximate removal of the Doppler shift inherently assumes a toroidal mode number of $n=1$ and can be a source of error if the minimum observed toroidal mode number is greater than $n=1$. The error associated with this, however, is expected to be less than the peak rotation observed at mid radius which is typically less than 

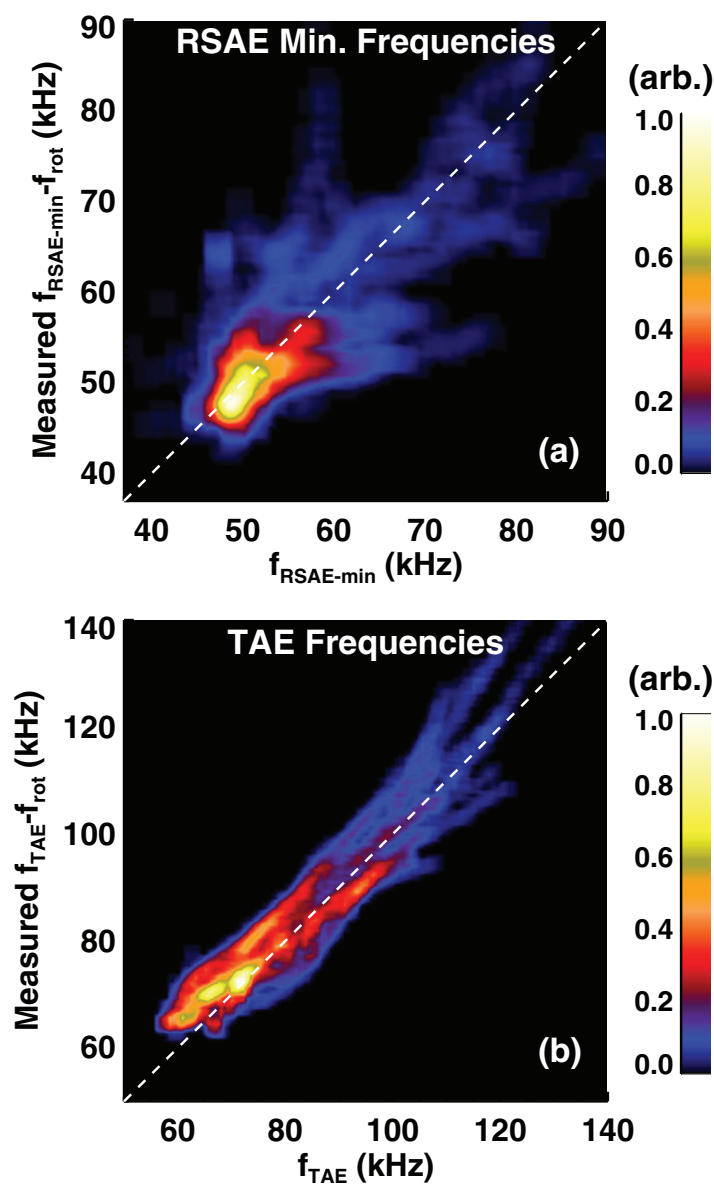

Figure 6. (a) Measured RSAE minimum frequencies versus those predicted by equation (3). (b) Measured TAE frequencies versus those predicted by equation (1). Data for both $(a)$ and $(b)$ obtained from 38 separate discharges in the range $t=300-1000 \mathrm{~ms}$. Note, measured frequencies have rotation frequency subtracted.

$10 \mathrm{kHz}$ and would always act to decrease the measured frequencies in figures $6(a)$ and $(b)$.

\section{ECH impact on AEs for a range of conditions and interpretation}

Figure 7(a) shows a spectrogram for the 'ECH near $q_{\text {min }}$ ' discharge 157735 presented in figure 4(c), except over a longer time window. In this figure, after approximately $t=650 \mathrm{~ms}$, the activity shifts from primarily TAEs to a mix of RSAEs then essentially only RSAEs, despite deposition of ECH near mid-radius. The model RSAE spectra are shown overlaid on the spectrogram in figure $7(b)$ along with $f_{\mathrm{GAM}}, f_{\mathrm{RSAE}-\mathrm{min}}$ and $f_{\text {TAE }}$. Before $t \approx 600 \mathrm{~ms}, f_{\text {RSAE-min }}$ is near or above $f_{\text {TAE }}$ and no frequency sweeping is predicted. After $t \approx 600 \mathrm{~ms}$, however, $f_{\mathrm{RSAE}-\mathrm{min}}$ drops precipitously and more typical frequency sweeping RSAEs are expected. This type of behavior is consistent with the beta suppression mechanism for RSAEs discussed by Fredrickson [32], with the exception that the model here includes the additional gradient upshift of the RSAE minimum frequency. An interesting question is what causes the rapid drop in $f_{\mathrm{GAM}}$ and $f_{\mathrm{RSAE}-\mathrm{min}}$. The dominant kinetic profile that is changing over this interval is electron temperature, time
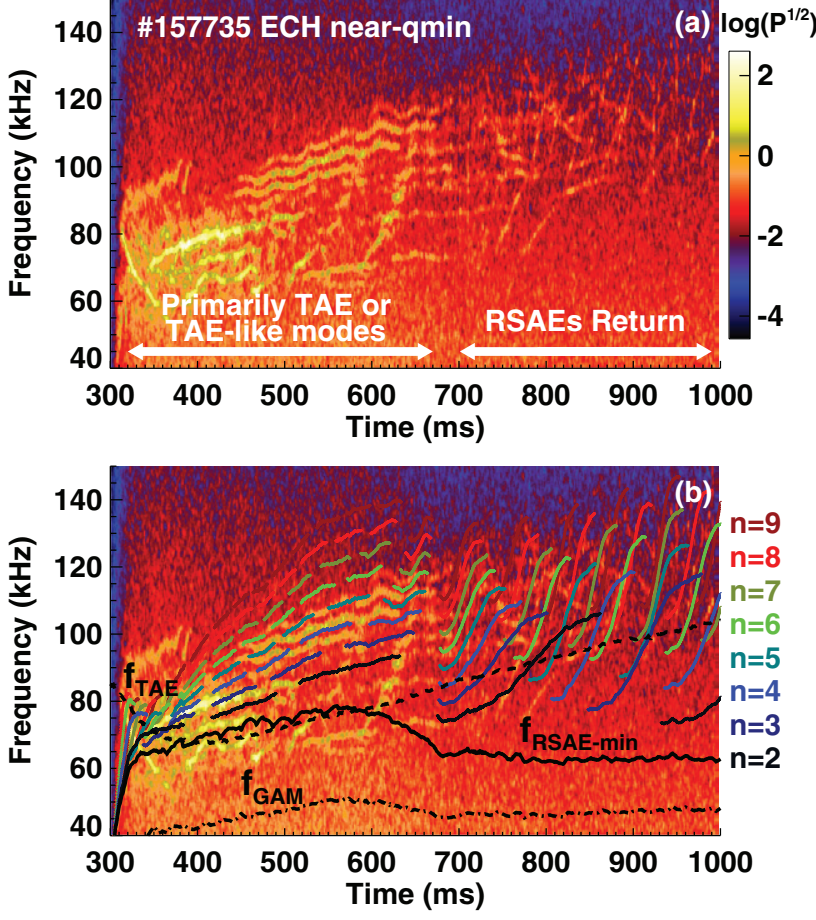

Figure 7. (a) Combined ECE spectrogram for discharge 157735 with ECH heating near $q_{\text {min }}$ (b) Model for RSAE frequency evolution overlaid on same spectrogram from panel $(a)$. Both ECH and NBI heating begin at $t=300 \mathrm{~ms}$ and extend over window shown.

histories of which at different radii are shown in figure 8(a) along with the injected neutral beam and ECH power waveforms in figure $8(b)$. Despite the fact that the heating is held essentially constant, a rapid drop in $T_{\mathrm{e}}$ occurs. It is this drop in $T_{\mathrm{e}}$ that causes a shift in the AE activity and a return to frequency sweeping RSAEs. The exact cause of the rapid drop in $T_{\mathrm{e}}$ is under investigation but one intriguing possible explanation is that a rapid increase in transport of beam ions brought about by the series of modes beginning at $t \approx 600 \mathrm{~ms}$ causes a reduction in central neutral beam heating efficiency. It is pointed out that in this interval, strongly driven TAEs are dominant which are actually thought to be typically more effective than RSAEs at causing EP transport due to their higher poloidal harmonic content and potentially larger radial extent, often extending to the plasma edge in DIII-D plasmas [35, 46, 56-58]. Indeed, previous experiments with ECH 'stabilized' RSAEs but a remaining spectrum of TAEs still showed neutron deficits of greater than $60 \%$ [1]. Recent experiments aimed at utilizing the FIDA modulation approach [59] and ECH to preferentially excite RSAEs or TAEs with the goal of quantifying the differences in EP transport have been performed and will be the subject of a future publication [60].

Figure 9 shows that a relatively minor delay in ECH injection turn-on (switched to $t=500 \mathrm{~ms}$ from $t=300 \mathrm{~ms}$ ) causes a radical change in the AE spectrum from that in figure 7. A $200 \mathrm{~ms}$ delay in the ECH turn-on time causes the AE spectrum to shift from TAE dominated to RSAE dominated and in fact, when ECH is turned on, the RSAEs appear to become stronger. This result clearly shows ECH near $q_{\text {min }}$ does not 

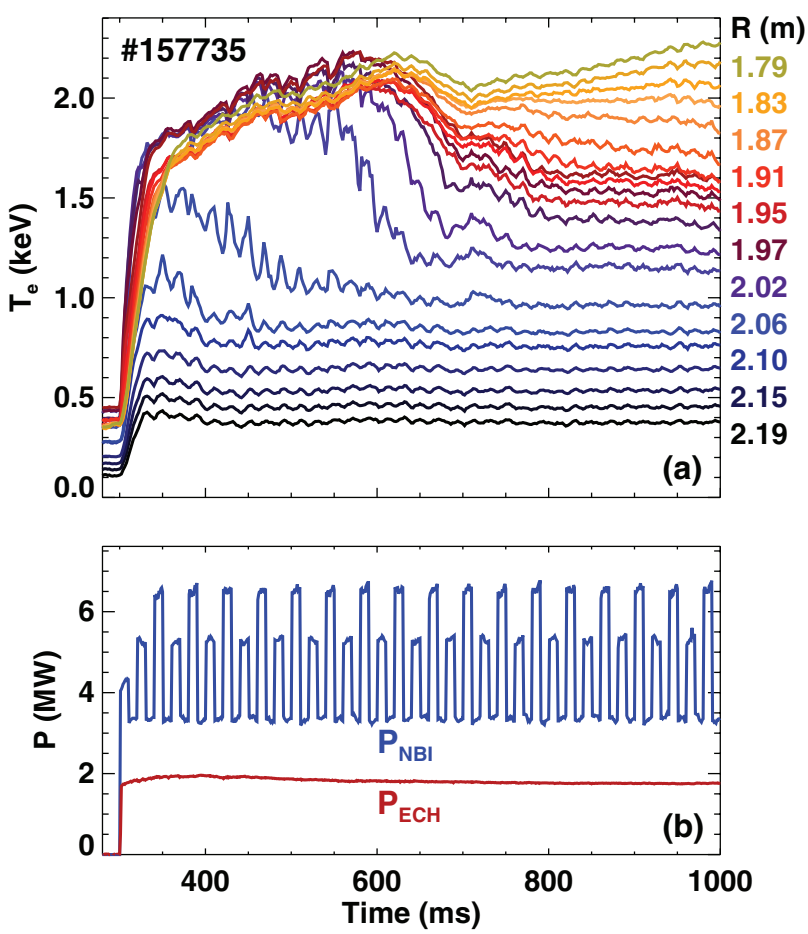

Figure 8. Discharge 157735. (a) ECE measured electron temperature evolution at several different radii across outboard midplane. Colors correspond to major radii listed on right. $(b)$ Temporal evolution of total neutral beam heating power $\left(P_{\mathrm{NBI}}\right)$ and electron cyclotron heating power $\left(P_{\mathrm{ECH}}\right)$.

automatically mean RSAEs will be suppressed. When one looks at the model prediction for this case (figure $9(b)$ ), $f_{\text {RSAE-min }}$ is well below $f_{\text {TAE }}$ over the entire current ramp and the predicted frequencies agree extremely well with those observed. Another interesting change in this discharge is the appearance of more downsweeping RSAEs [28, 61], a feature which is not as common as the up-sweeping RSAE.

Spectra from a set of discharges with similarly unexpected results are shown in figure 10; in these discharges, the same ECH steering geometry and timing as figures $4(b)$ and $(c)$ was used but the current ramp rate was reduced from $0.8 \mathrm{MA} \mathrm{s}^{-1}$ to $0.44 \mathrm{MA} \mathrm{s}^{-1}$. By reducing the current ramp rate, the impact of the $\mathrm{ECH}$ was essentially reversed relative to that in figures 4(b) and (c). In discharge 157747 with ECH near the magnetic axis (figure 10(c)), the discharge is almost completely dominated by TAE-like modes, very little if any RSAE activity is apparent. For the slow current ramp ECH near $q_{\text {min }}$ discharge (figure $10(a)$ ), there is a brief period $(t \approx 450$ $-600 \mathrm{~ms}$ ) where frequency sweeping RSAEs are not clearly observed but it quickly returns to a case with a mix of RSAEs and TAEs. In both cases, motional Stark effect (MSE) data shows the safety factor profiles are reversed throughout the entire time windows shown. The model predictions for these discharges are given in figures $10(b)$ and $(d)$ where excellent agreement with the measured behavior is shown. For the case with ECH near $q_{\text {min }}$, a brief period exists where $f_{\text {RSAE-min }}$ is near $f_{\text {TAE }}$ and that period agrees well with the period of the discharge in which no frequency sweeping RSAEs are observed. Immediately following that period $(t \approx 600 \mathrm{~ms})$,
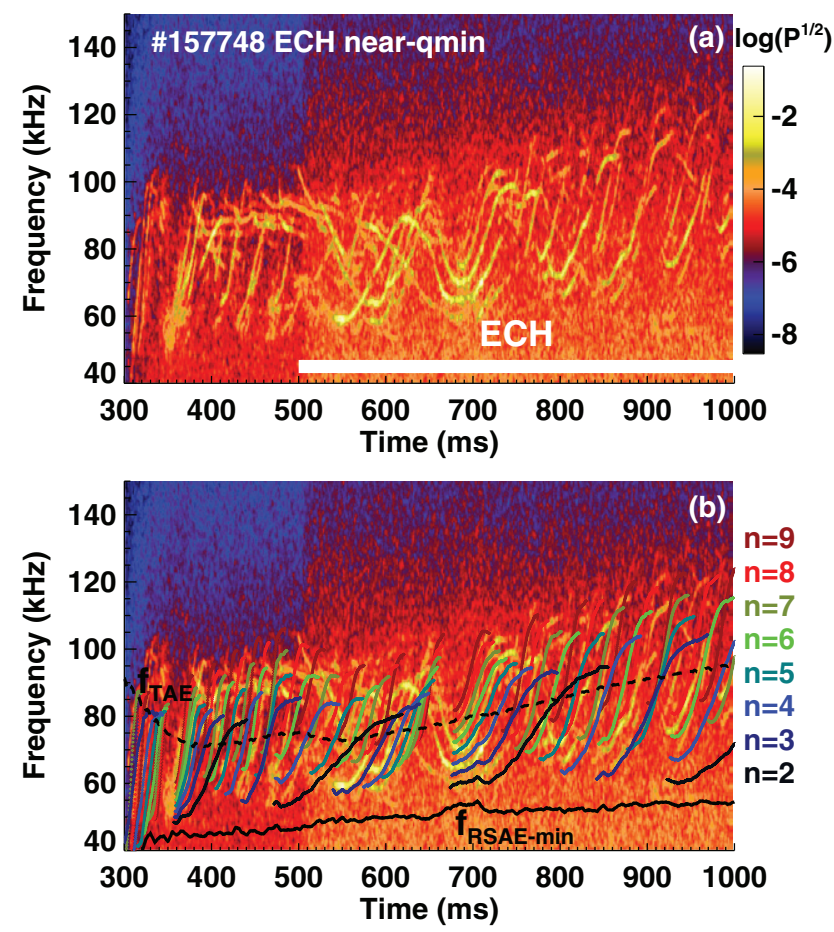

Figure 9. (a) Combined ECE spectrogram for discharge 157748 with ECH heating near $q_{\text {min }}$ (b) Model for RSAE frequency evolution overlaid on the same spectrogram from panel $(a)$. NBI heating begins at $t=300 \mathrm{~ms}$ and ECH heating begins at $t=500 \mathrm{~ms}$.

RSAEs return, and this behavior is captured by the model. It is conjectured that a similar effect to that in figure $8(a)$ must be occurring in this discharge that causes a rapid drop in $T_{\mathrm{e}}$ and consequently in $f_{\text {RSAE-min }}$. The predicted RSAE minimum frequency in discharge 157747, with ECH near the axis, on the other hand, remains elevated throughout the entire window shown and frequency sweeping RSAEs are not predicted or observed. The model again appears to capture the shift from frequency sweeping RSAEs to TAEs.

In the examples shown and essentially all other cases investigated $(38+$ discharges), the picture that RSAEs are observed only when $f_{\text {RSAE-min }}<f_{\text {TAE }}$ is consistent with the measured spectra. This statement is quantified in figure 11, where the predicted $f_{\text {RSAE-min }}$ is plotted versus $f_{\mathrm{TAE}}$ for several points during the current ramp portion of all discharges where AEs were observed. The points are color coded according to whether typical RSAEs were observed (RED) or not observed (BLACK). Overplotted is also where $f_{\text {RSAE-min }}=f_{\text {TAE }}$. In figure $11(b)$, the data are also histogrammed versus the ratio $f_{\text {RSAE-min }} / f_{\text {TAE }}$. A clear division exists between these two datasets; when $f_{\text {RSAE-min }} \ll f_{\text {TAE }}$ typical RSAEs are observed, while for $f_{\text {RSAE-min }} \lesssim f_{\text {TAE }}$ a mix of cases exists and for $f_{\text {RSAE-min }}>f_{\text {TAE }}$ typical frequency sweeping RSAEs are not observed.

\section{NOVA and TAEFL simulations}

The analysis presented in the previous sections shows ECH modification of $f_{\mathrm{GAM}}$ and $f_{\mathrm{RSAE}-m i n}$ through changes in $T_{\mathrm{e}}$ are likely causes for the change from typical frequency sweeping 

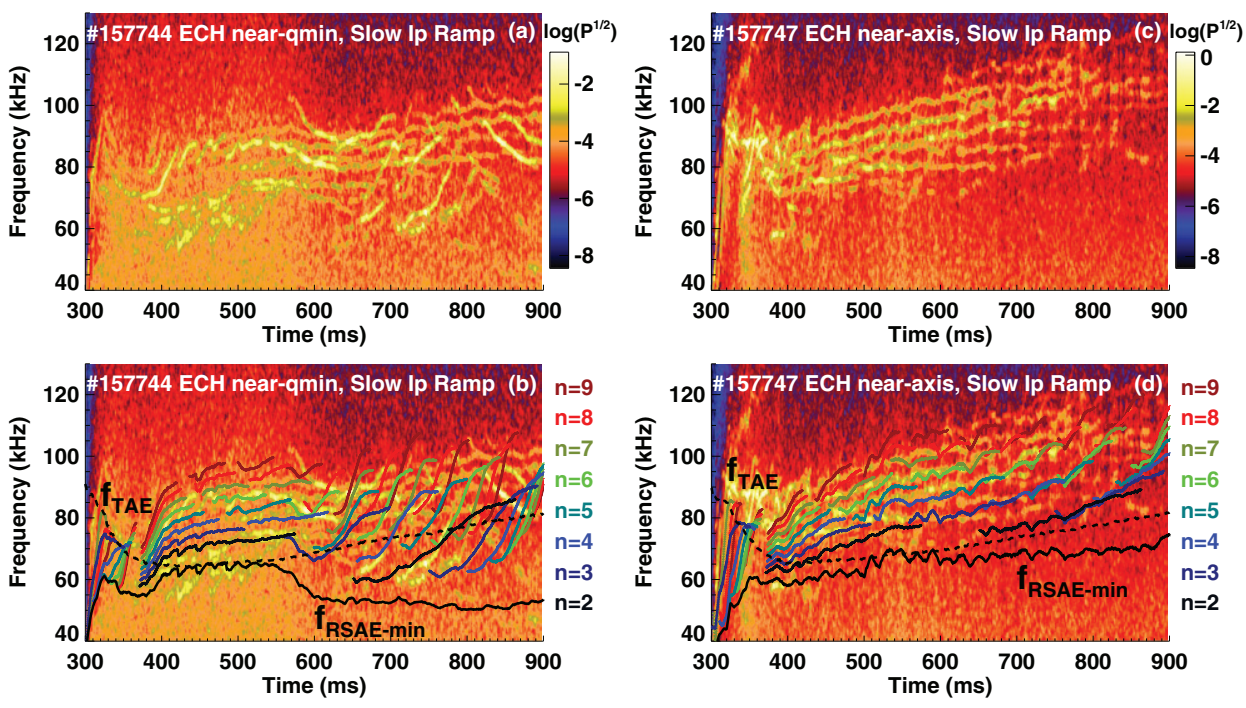

Figure 10. Current ramp rate reduced from $0.8 \mathrm{MA} \mathrm{s}^{-1}$ to $0.44 \mathrm{MA} \mathrm{s}^{-1}$ for both discharges shown. (a) Combined ECE spectrogram for discharge 157744 with ECH heating near $q_{\min }$. $(b)$ Model for RSAE frequency evolution overlaid on the same spectrogram from panel $(a)$. (c) Combined ECE spectrogram for discharge 157747 with ECH heating near the magnetic axis. (d) Model for RSAE frequency evolution overlaid on the same spectrogram from panel $(c)$. Both ECH and NBI heating begin at $t=300 \mathrm{~ms}$ and extend over the window shown.

RSAEs to a spectrum of TAEs or TAE-like modes in the range of discharges investigated; however, more rigorous calculations are required to determine the impact on the eigenmodes themselves and their stability. In this section the NOVA $[42,43]$ and TAEFL [44] codes are used to address these issues and provide additional insight into the impact of $\mathrm{ECH}$ on RSAEs. NOVA is an ideal MHD eigenvalue solver and TAEFL is non-perturbative gyrofluid code that can be run as initial value or eigenvalue solver. Both of these codes have successfully modeled AEs in many DIII-D plasmas [2, 37, 45, 46], as well as other devices including JT60 [62], TFTR [63], JET [28], NSTX [64], CMOD [29] and ASDEX-Upgrade [50].

\subsection{NOVA simulations}

NOVA simulations have been carried out for two of the discharges discussed above, the first is 157737 , the 'NO ECH' case presented in figure 4(a), and the second is 157735 , the 'ECH near $q_{\text {min }}$ ' case (figure $4(c)$ ), both near $q_{\min }=4$. The rationale for the study that follows is similar to the approach taken in the actual experiment, i.e. to start with a very well understood case that has been modeled and looked at with success in the past then, to mimic changes in pressure brought about by central ECH, make variations in equilibrium pressure (NOVA is a single fluid MHD code) and see if we can reproduce the types of changes in RSAEs observed in the previous sections. We will then move to the $\mathrm{ECH}$ case, which has not been analyzed satisfactorily in the past, and show that a similar change in RSAE activity to that found experimentally is predicted by the NOVA simulations.

The experimental profiles used as inputs for these two cases are shown in figure 3 . As mentioned, the primary differences in these two cases are in the $q$-profile (figure 3(a)) and electron temperature profile (figure $3(b)$ ). For ECH near $q_{\min }$, the central safety factor is elevated relative to the no ECH case. This is understandable since we are looking during the
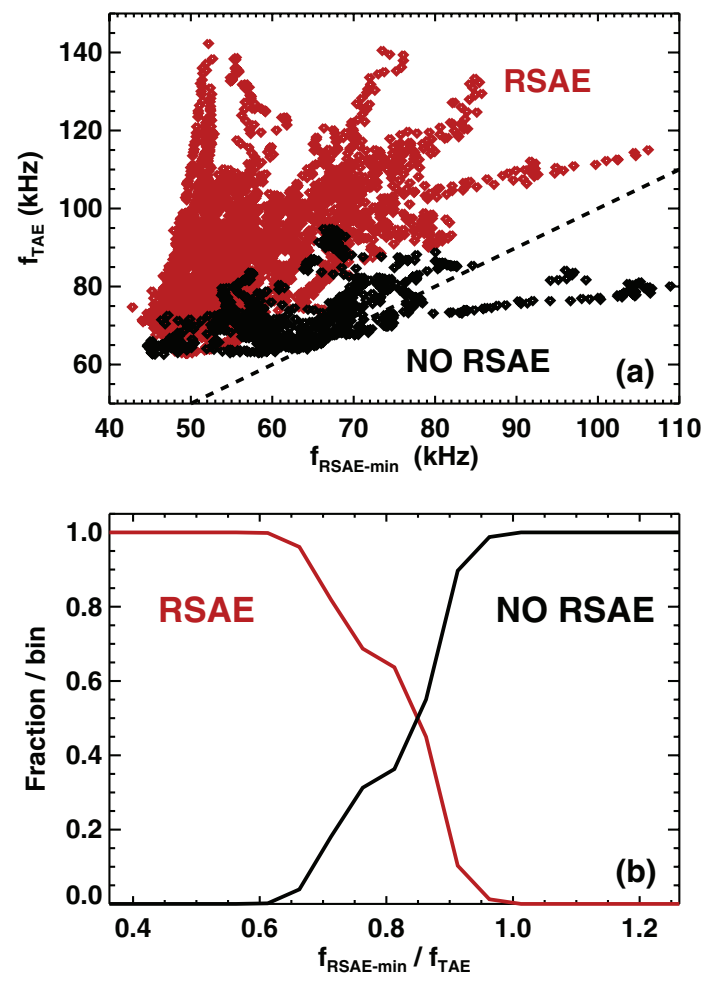

Figure 11. Data obtained from 38 separate discharges in the range $t=300-1000 \mathrm{~ms}$. Points considered have $P_{\mathrm{NBI}}>2 \mathrm{MW}$ to ensure sufficient AE drive is available. (a) Points indicate $f_{\mathrm{RSAE}-m i n}$ and $f_{\text {TAE }}$ using experimental kinetic profiles and color coding indicates if typical frequency sweeping RSAEs were observed (RED) or no frequency sweeping RSAEs were observed (BLACK). Overlaid dashed line indicates $f_{\text {TAE }}=f_{\text {RSAE-min }}$ ( $b$ ) Data from panel $(a)$ histogrammed versus the ratio $f_{\mathrm{RSAE}-m i n} / f_{\mathrm{TAE}}$ showing what fraction has observable RSAE activity or none.

current ramp portion of the discharge and the higher central electron temperature, due to heating, causes a delay in the current penetration to the axis. It is noted that in both cases, 


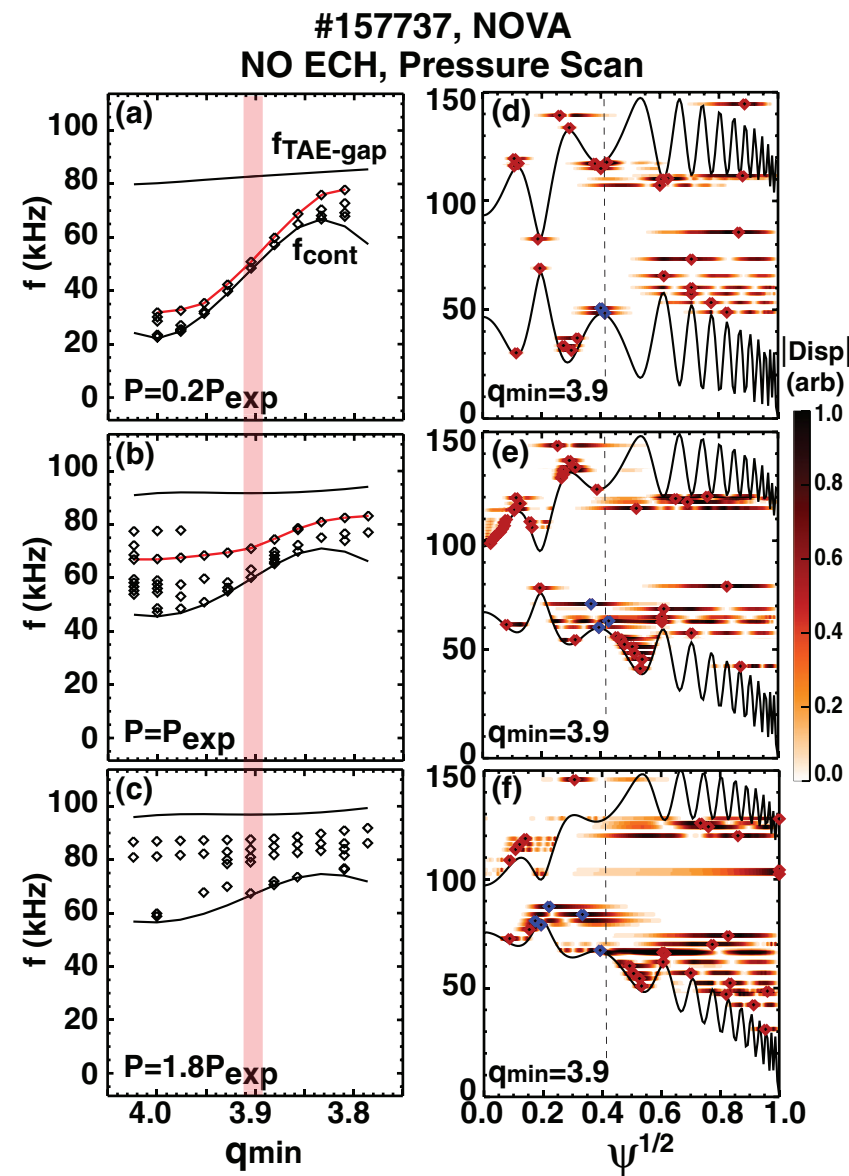

Figure 12. $n=3$ NOVA simulations for discharge 157737 with equilibrium pressure scaled to $(a)$ and $(d) P=0.2 P_{\exp }$, (b) and (e) $P=P_{\text {exp }}$, and $(c)$ and $(f) P=1.8 P_{\text {exp }}$ (a) $-(c)$ show 'RSAE' frequencies with the thin red line indicating fundamental RSAE. Solid black curves indicate continuum tip and center of TAE gap at $q_{\min }$. Red bar indicates $q_{\min }$ that continua in panels $(d)-(f)$ are shown for. Panels $(d)-(f)$ have contours overlaid indicating eigenmode frequencies and displacement radial structure. Diamond markers indicate mode peak locations with blue indicating modes that fulfill criteria defined for RSAEs and red all other modes.

Dashed vertical line indicates radial location of $q_{\min }$.

the fast ion pressure is comparable to the thermal pressure (figure $3(d)$ ), this is included only approximately in NOVA by use of the total equilibrium pressure in the calculations. TAEFL, however includes the fast ion population non-perturbatively as a separate species which is more rigorous. In each case investigated (i.e. with and without $\mathrm{ECH}$ ), the actual fast ion pressure profile is similar, with the major differences to the total pressure being due to differences in the thermal pressure.

Several of the following figures are structured in a similar way so a general description will be given before stepping through each. NOVA is a linear eigenvalue solver that finds all eigenmodes for a given $n$ in a given frequency range. For each case investigated, the minimum safety factor was scanned from $q_{\min }=4.02$ to $q_{\min }=3.79$ in order to resolve a full $n=3$ RSAE frequency sweep. $n=3$ is chosen as the focus because, experimentally, $n=3$ is typically the strongest mode observed. For simplicity, toroidal rotation was not included in these calculations. NOVA eigenmodes can be

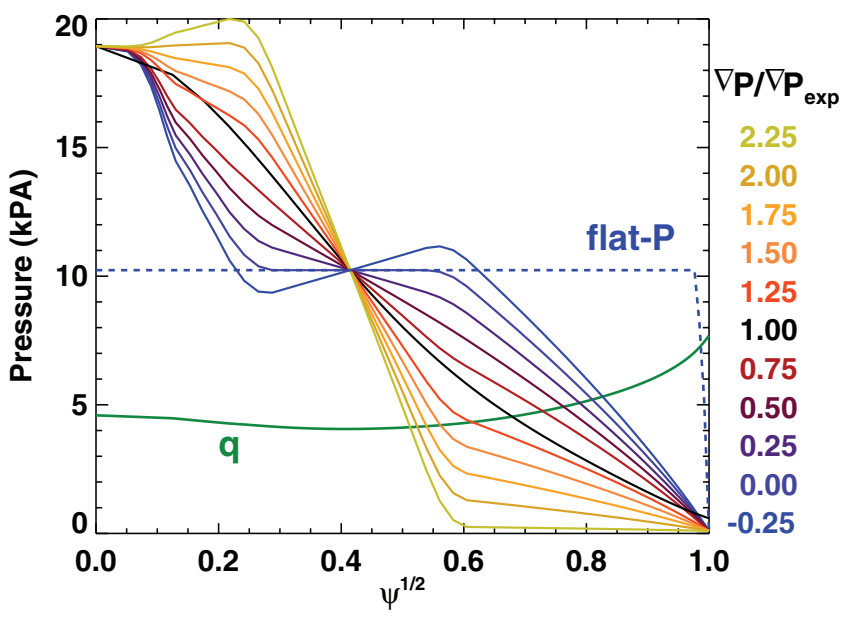

Figure 13. Variety of pressure profile variations used in NOVA simulations of discharge 157737. Colors indicate pressure gradient at $q_{\min }$ relative to the experimental pressure gradient at $q_{\min }$. Also overlaid is a dashed line representing the flat pressure profile used and a solid green line which is the safety factor $(q)$ profile.

be a mix of TAEs, RSAEs, beta induced Alfvén eigenmodes $[65,66]$ (BAEs), etc. To help discriminate, an RSAE filter was created that searches through the eigenmodes and finds all modes that are likely RSAEs. For the purposes of this search, RSAEs were identified according to the following criteria: localization near $q_{\min },>25 \%$ of the total displacement in the $m=n q_{\text {min }}$ harmonic, frequency above the continuum tip at $q_{\min }$, and frequency below the center of the TAE gap. The poloidal harmonic content criteria allows for the typical RSAE with one dominant poloidal harmonic $(m)$ at $q_{\text {min }}=m / n$ transitioning to one with two dominant poloidal harmonics ( $m$ and $m-1)$ as $q_{\min }$ approaches $(m-1 / 2) / n$ to be identified as well as RSAEs that are linearly coupled to TAEs [46]. In figures $12,14,15$ and 17 , the right column represents $n=3$ continua for a fixed $q_{\min }$ with a contour plot showing mode displacements versus square root of normalized poloidal flux $\left(\psi^{1 / 2}\right)$ and frequency. A diamond marks the peak displacement location of each mode where blue is used for RSAE-like modes and red represents all other modes. The left column of each figure displays the frequencies of all modes fitting the RSAE filtering criteria versus $q_{\min }$. Where the fundamental RSAE can be identified, its frequency evolution versus $q_{\min }$ is followed by a red line. On the same plots, the continuum frequency at $q_{\min }$ and at the center of the TAE gap are overlaid as solid black lines. Here, the term fundamental RSAE refers to the highest frequency RSAE with no radial nodes in its eigenmode amplitude envelope [2, 26, 67]. RSAEs and TAEs are anti-sturmian so modes below those connected by the red line are higher radial harmonics having successively lower frequencies. Higher radial harmonics can be identified on the right column continuum plots by looking for nodes in the radial structures shown. Experimentally, the appearance of higher radial harmonic RSAEs is common $[2,49,67]$ and often observed more frequently at higher pressures. It is noted that although NOVA often finds several radial harmonics, in experiment typically only the fundamental or first radial harmonic RSAE are observed. Overlaid on each of 
\#157737, NOVA

NO ECH, Flat Pressure Profile Scan
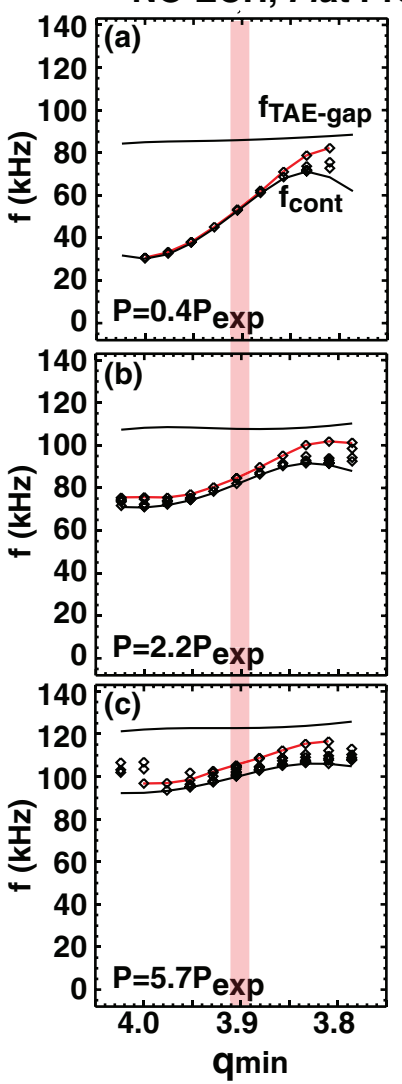
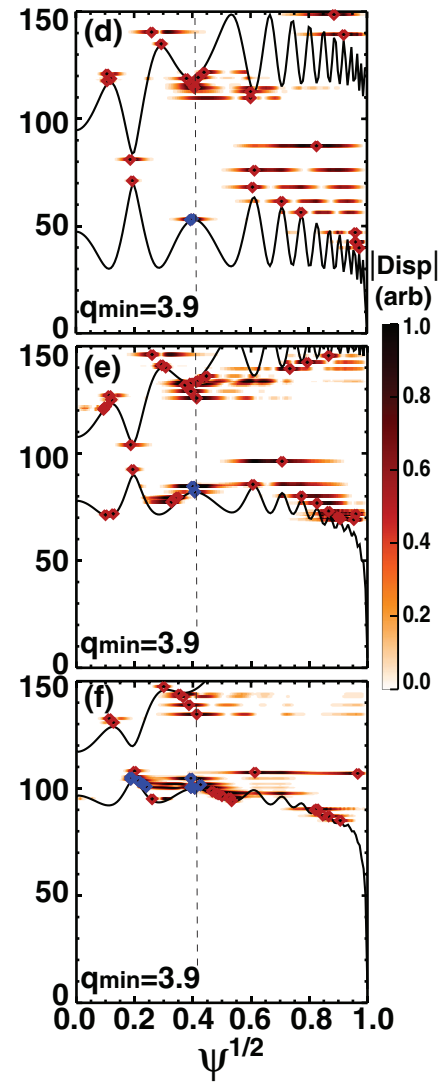

Figure 14. $n=3$ NOVA simulations for discharge 157737 with a flat pressure profile scaled so the pressure relative to the experimental pressure at $q_{\min }$ is $(a)$ and $(d) P=0.4 P_{\text {exp }},(b)$ and (e) $P=2.2 P_{\text {exp }}$, and $(c)$ and $(f) P=5.7 P_{\text {exp }}$. $(a)-(c)$ show 'RSAE' frequencies with the thin red line indicating fundamental RSAE. Solid black curves indicate continuum tip and center of TAE gap at $q_{\min }$. Red bar indicates $q_{\min }$ that continua in panels $(d)-(f)$ are shown for. Panels $(d)-(f)$ have contours overlaid indicating eigenmode frequencies and displacement radial structure. Diamond markers indicate mode peak locations with blue indicating modes that fulfill criteria defined for RSAEs and red all other modes. Dashed vertical line indicates radial location of $q_{\mathrm{min}}$.

the left columns is a thick red bar that identifies the particular $q_{\min }$ value for which the continua and individual mode structures are shown in the right column of each figure.

Shown in figure 12 are $n=3$ NOVA calculations for discharge 157737, the 'NO ECH' case, in which the pressure profile has been scaled above and below the actual experimental values $\left(P_{\text {exp }}\right)$. The figure shows results for $0.2 P_{\exp }, P_{\text {exp }}$, and $1.8 P_{\text {exp }}$, however, calculations were actually carried out from zero to $3 \times$ that in experiment to help identify the changes with individual modes and to track the fundamental RSAE more clearly. For the $0.2 P_{\exp }$ case shown in figures $12(a)$ and $(d)$, a very clear fundamental RSAE is identified as well as a radial harmonic. Both modes are peaked very near $q_{\min }$. To aid the eye, overlaid on each contour in figures $12(d)-(f)$ is a thin vertical dashed line indicating the location of $q_{\min }$. This line helps identify an important shift in the RSAE eigenfunctions that occurs as the central pressure is increased-the peak of the RSAE moves inward and away from $q_{\text {min }}$, a behavior

\#157737, NOVA

NO ECH, Pressure Grad. Scan
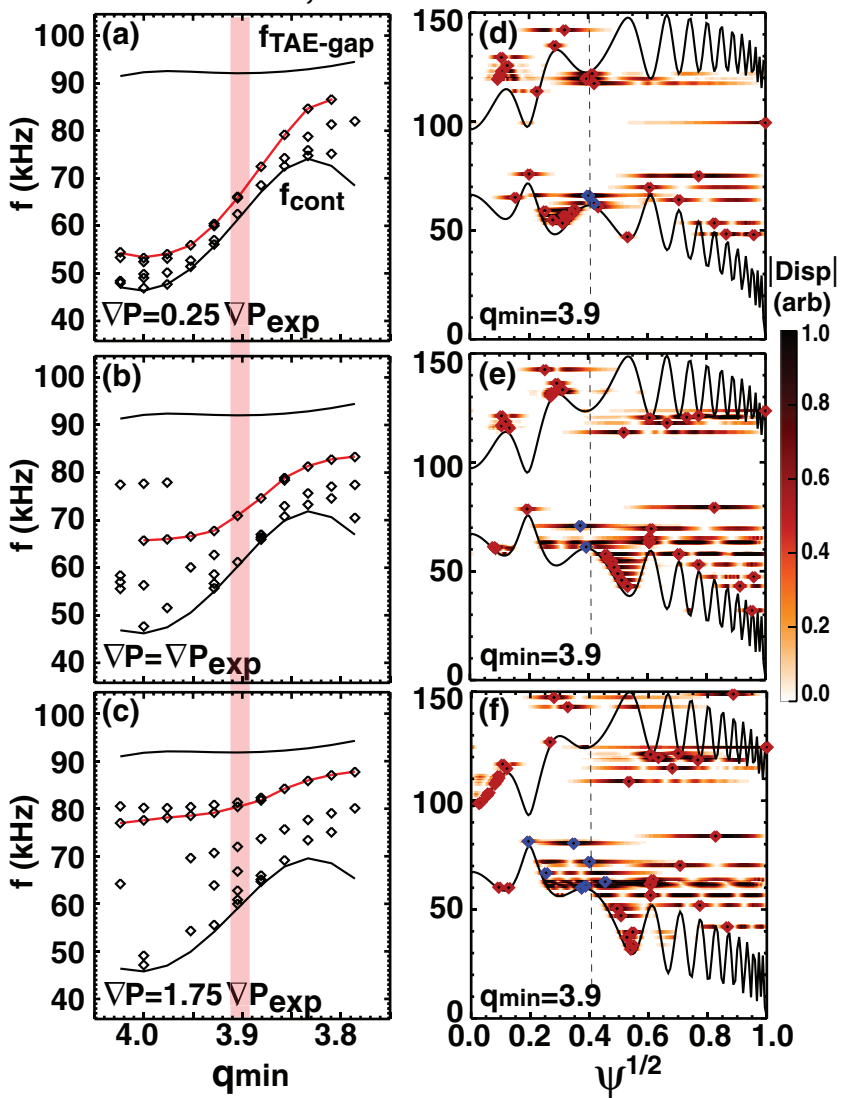

Figure 15. $n=3$ NOVA simulations for discharge 157737 with the pressure profile modified such that the gradient at $q_{\min }$ relative to the experimental value at $q_{\min }$ is: $(a)$ and $(d) \nabla P=0.25 \nabla P_{\text {exp }},(b)$ and $(e) \nabla P=\nabla P_{\text {exp }}$, and $(c)$ and $(f) \nabla P=1.75 \nabla P_{\text {exp. }}(a)-(c)$ show 'RSAE' frequencies with the thin red line indicating fundamental RSAE. Solid black curves indicate continuum tip and center of TAE gap at $q_{\text {min }}$. Red bar indicates $q_{\min }$ that continua in panels $(d)-(f)$ are shown for. Panels $(d)-(f)$ have contours overlaid indicating eigenmode frequencies and displacement radial structure. Diamond markers indicate mode peak locations with blue indicating modes that fulfill criteria defined for RSAEs and red all other modes. Dashed vertical line indicates radial location of $q_{\min }$.

which can be seen for the higher pressure cases in figure 12 . For the actual experimental profiles (figures 12(b) and (e)), the fundamental RSAE is also clearly identified and its frequency sweep is very similar to that observed experimentally. As expected, as the pressure is increased the RSAE frequency, for a given $q_{\mathrm{min}}$, increases as does $f_{\mathrm{GAM}}$ and the width of the lowest gap. The frequency separation of the RSAE from the continuum tip at $q_{\min }$ also increases, an effect which is predominantly due to an increase in the pressure gradient and $f_{\nabla}$. Additionally, several radial harmonic RSAEs are present and all of their frequencies are further separated from the continuum more than the lower pressure case. The RSAE eigenfunction peaks are also found to be displaced inward from $q_{\min }$ as can be seen in figure 12(e). It will be shown later that this result is due to an increase in the equilibrium pressure gradient. At only $1.8 \times$ the experimental pressure (figures 12(c) and $(f)$ ), the RSAE frequencies are even further from the continuum tip at $q_{\min }$ and the fundamental RSAE is 
hard to identify, undergoes very little frequency sweeping and is likely coupled to the core TAE just above the lower continuum at $\psi^{1 / 2} \approx 0.2$ (clearly observeable in figures $12(d)$ and (e)). The fundamental RSAE has not been labelled because a determination is difficult but it is thought the upper set of diamonds in figures 12(c) correspond to its frequency evolution. The RSAEs for this case are all pushed significantly inward, do not sweep in frequency significantly and are coupled to core TAEs - they are very different from the standard RSAE.

Scaling the experimental pressure profile, as was done in figure 12, is useful but it has the effect of changing both the plasma pressure and pressure gradient at $q_{\text {min }}$. Both are known to be important to the RSAE existence, frequency and structure [23-27, 30]. To separate these effects, two separate scans were carried out. The first uses a flat pressure profile rigidly scaled from 0-7 $\times$ the pressure at $q_{\text {min }}$. The second scan held the pressure at $q_{\min }$ fixed but rigidly varied the pressure gradient about $q_{\min }$. For the pressure gradient scan, the gradient was held constant over a radial width approximately the distance between the $(m+1) / n$ rational surfaces surrounding $q_{\text {min }}$. The profiles employed in this scan are shown in figure 13 along with one of the flat pressure profiles.

Figure 14 shows NOVA results for three cases from the flat pressure profile scan, $P=0.4 \times, 2.2 \times$ and $5.7 \times$ the experimental pressure at $q_{\text {min }}$. Perhaps the most obvious change as the pressure is increased in this scan is the upshift in the lower continuum as well as a smaller but significant increase in the center of the TAE gap. Both of these effects are to be expected. In all cases, a prototypical fundamental RSAE was found for almost the entirety of the $q$ range investigated and the fundamental RSAEs are localized and peaked almost exactly at $q_{\text {min }}$. The frequency separation from the continuum tip is also much smaller than the experimental case shown in figure 12(b), particularly for the lower pressure cases shown in figures 14(a) and $(b)$. It is also pointed out that for flat pressure profiles with $P_{q \text { min }}$ at the experimental value or lower, higher radial harmonic RSAEs are not found as clearly, except near the top of the frequency sweep.

Figure 15 shows NOVA results for three cases from the pressure gradient scan, $\nabla P=0.25 \times, 1.0 \times$ and $1.75 \times$ the experimental pressure gradient at $q_{\text {min }}$. Unlike the pressure scan cases, the continuum frequencies at $q_{\min }$ are essentially unchanged throughout the entire pressure gradient scan range. The RSAE frequencies, however, are radically different and the frequency sweep range is much reduced. For instance, the fundamental RSAE frequency at $q_{\min }=4$ varies from $53 \mathrm{kHz}$ to $78 \mathrm{kHz}$ as the gradient is changed from 0.25 to $1.75 \times \nabla P_{\exp }$, while the upper end of the frequency sweep range remains similar. The other obvious difference is the eigenmodes are shifted radially inward. This can be seen by looking at figures $15(d)$ and $(f)$. Apparently, the RSAE potential well is made asymmetric by the addition of a pressure gradient and this causes a shift in the mode localization. Depending on the concomitant upshift in frequency, this effect can serve to drive the mode radially into the continuum, an effect which is potentially exacerbated by the large magnetic shear inside of $q_{\text {min }}$. In terms of getting $f_{\mathrm{RSAE}-\mathrm{min}}$ closer to $f_{\mathrm{TAE}}$, pressure gradient changes appear to be very effective since they do not
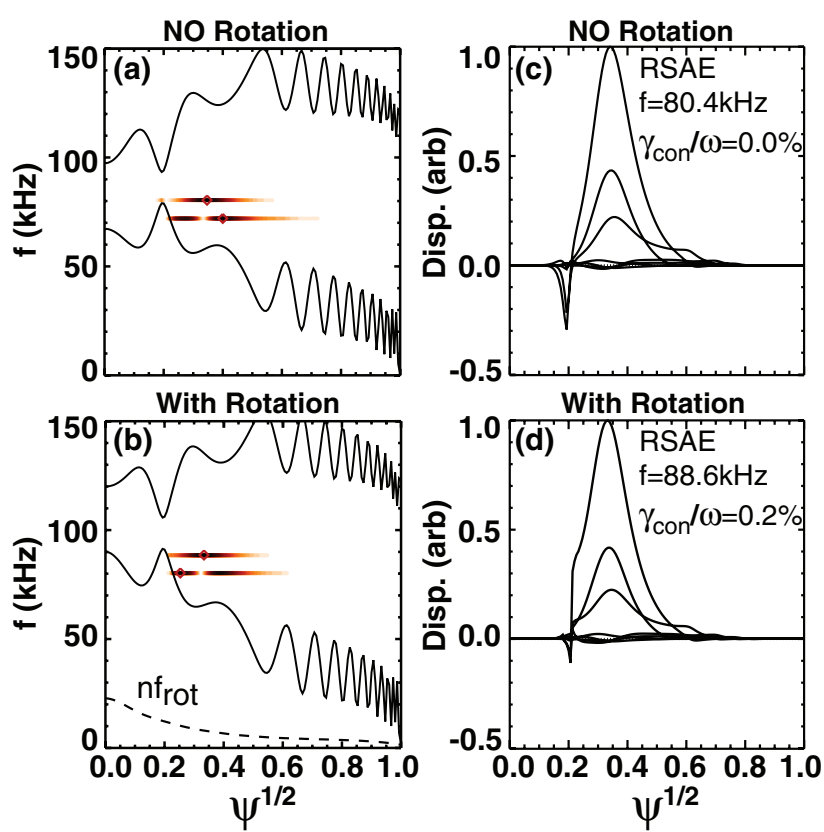

Figure 16. $n=3$ NOVA simulations for the case in figure $15(f)$ without $((a)$ and $(c))$ and with $((b)$ and $(d))$ toroidal rotation included. Panels $(a)$ and $(b)$ show the $n=3$ continuum along with the fundamental and next radial harmonic RSAE frequencies and eigenfunction extent overlaid. The Doppler shift for the $n=3$ continuum is over plotted in $(b)$ as a dashed line. The displacement color scale is the same as shown in figure 15. Panels $(c)$ and (d) show the detailed radial structure of the different poloidal harmonics ( $m=9-15)$ for the fundamental RSAE along with the continuum damping $\gamma_{\text {con }} / \omega$.

shift the gap noticeably but upshift the RSAE and additionally, can drive the modes into the continuum radially as well.

Another important parameter which can enhance the impact of the ECH induced pressure gradient shift in RSAE localization, discussed above, is the presence of toroidal rotation and, specifically, shear in the toroidal rotation profile. While the toroidal rotation profiles in the L-mode discharges discussed here are predominantly determined by the beam injection, fast ion transport or even spontaneous changes due to ECH modification of equilibrium parameters also contribute. With rotational shear, a radial shift of the eigenmode can result in increased interaction with the Alfvén continuum and enhanced damping. This effect has been discussed in detail in [15] and [16]. In figure 16, the impact of toroidal rotation on the $n=3$ RSAEs of figure $15(f)$ is investigated. Figures 16(a) and $(b)$ show the gap structure with and without toroidal rotation respectively along with the amplitude of the fundamental and first radial harmonic RSAE overlaid. Both modes experience more interaction with the continuum as a result of the sheared rotation profile (the Doppler shift, $n f_{\text {rot }}$, is given as a dashed line in panel figure 16(b)). The impact on the fundamental RSAE is found by comparing the radial structures and continuum damping for each case, shown in figures $16(c)$ and $(d)$. Without toroidal rotation, minor interaction with the continuum occurs and the calculated continuum damping is negligibly small, i.e. $\gamma_{\text {con }} / \omega \approx 0.0$. With toroidal rotation included, the mode interacts more strongly with 


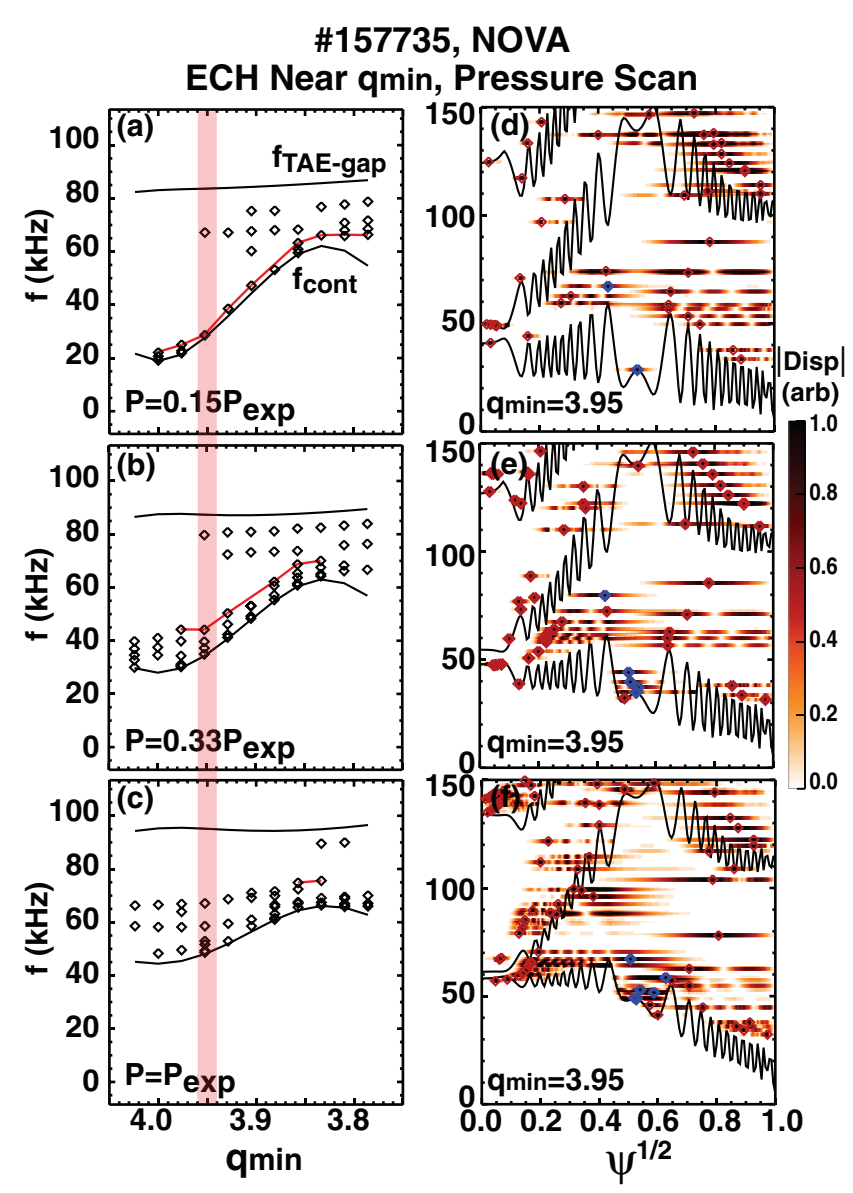

Figure 17. $n=3$ NOVA simulations for discharge 157735 'ECH near $q_{\text {min }}$ ' with equilibrium pressure scaled to $(a)$ and $(d)$ $P=0.15 P_{\text {exp }},(b)$ and $(e) P=0.33 P_{\text {exp }}$, and $(c)$ and $(f) P=P_{\text {exp. }}$. $(a)-(c)$ show 'RSAE' frequencies with the thin red line indicating fundamental RSAE. Solid black curves indicate continuum tip and center of TAE gap at $q_{\min }$. Red bar indicates $q_{\min }$ that continua in panels $(d)-(f)$ are shown for. Panels $(d)-(f)$ have contours overlaid indicating eigenmode frequencies and displacement radial structure. Diamond markers indicate mode peak locations with blue indicating modes that fulfill criteria defined for RSAEs and red all other modes.

the continuum, its radial eigenfunction is cut off abruptly at $\psi^{1 / 2} \approx 0.21$, and the continuum damping $\gamma_{\text {con }} / \omega$ rises to $0.2 \%$.

Now moving to discharge 157735 , with ECH at $q_{\text {min }}$, we present results from a pressure scan similar to that in figure 12 . The goal of these calculations is to see if NOVA finds typical RSAEs for this scenario and to determine if the frequency sweeping RSAE is even an eigenmode of the experimental equilibrium. These results are shown in figure 17, where calculations for $P=0.15 \times, 0.33 \times$ and $1.0 \times$ the experimental pressure are given. One striking feature of the continua plots in the right hand column of figure 17 is the abundance of modes NOVA finds in the TAE gap, both inside and outside of $q_{\text {min }}$. It is conjectured that the abundance of TAEs inside of $q_{\min }$ is due to the high magnetic shear in this region. The fundamental RSAE was not found for the experimental pressure case, except for possibly a very short $q_{\min }$ range (figure $17(c)$ ), however when the pressure is scaled down significantly, the fundamental frequency sweeping RSAE can be identified as is shown in figures $17(a)$ and $(b)$. At even $0.33 \times$ the experimental pressure, the fundamental RSAE is pushed inward and is found to interact with the continuum. In this equilibrium, for the experimental pressure, the typical RSAE is not an eigenmode of the system, instead, it is pushed very far into the TAE gap and strongly coupled to the TAEs found inside of $q_{\text {min }}$. The resulting mode has a mix of TAE and RSAE-like properties, something that is explained in figure 18. In figure 18(a), the continuum for $q_{\min }=3.83$ at the experimental pressure is shown. The radial structure of the highlighted mode is plotted for several $q_{\text {min }}$ values in figures $18(b)$ and $(d)$. This mode does not sweep in frequency yet the poloidal harmonic content behaves exactly like that of the prototypical RSAE, i.e. it has one dominant poloidal harmonic $(m=12)$ at $q_{\min }=m / n$ and transitions to two dominant poloidal harmonics $(m=12$ and $m-1=11)$ as $q_{\min }$ approaches $(m-1 / 2) / n$. Further, these harmonics are peaked at the $q_{\min }$ surface. The TAE like features are the constant frequency and additional radial harmonics inside at $\psi^{1 / 2}<0.4$.

\subsection{TAEFL simulations}

To complement the NOVA analysis described in the previous section, TAEFL simulations which include fast ions nonperturbatively were carried out. For these simulations, TAEFL was run as an eigenvalue solver so it is capable of finding the spectrum of eigenmodes along with the growth rates. In addition to changes in the eigenmodes with $\mathrm{ECH}$, the TAEFL calculations help address the question of how the mode stability is changed.

TAEFL $n=3-6$ calculations for discharges 157737 'No ECH' and 157735 'ECH Near $q_{\text {min }}$ ' are shown in figure 19. For each discharge, all inputs are those given in figure 3 with the $q$-profile translated over the range $q_{\min }=4.3-3.7$. In figure $19(a)$, the simulations show a spectrum of RSAEs are expected with large growth rates $(\gamma / 2 \pi f>50 \%$ in some cases). This calculated spectrum is very similar to the measured spectrum shown in figure 5(a) and that of the $q$-evolution model in figure $5(b)$; note, the approximate experimental time range simulated is $t \approx 370-540 \mathrm{~ms}$. One difference between the TAEFL calculations in figure $19(a)$ and the experimental spectrum of modes, however, is that the TAEFL calculations predict downsweeping RSAEs over a larger $q_{\text {min }}$ range than observed experimentally. The exact reason for this discrepancy is unknown, however, as mentioned previously, the downsweeping RSAE is quite uncommon in DIII-D plasmas [61] and it is an area of active research to understand this effect and resolve the differences. One potential source for the difference in unstable modes predicted by TAEFL and those observed experimentally is the assumption of a Maxwellian fast ion population. The TAEFL results for discharge 157735 that ' $\mathrm{ECH}$ Near $q_{\text {min }}$ ' are shown in figure $19(b)$, where an extremely different spectrum of unstable modes is found as compared to the 'No ECH case'. For the ECH case, TAEFL finds no typical frequency sweeping RSAEs and instead finds modes that have a much reduced sweep range and growth rates that are reduced by approximately $70 \%$ or more compared to those found in figure $19(a)$. These modes are a combination of higher radial 


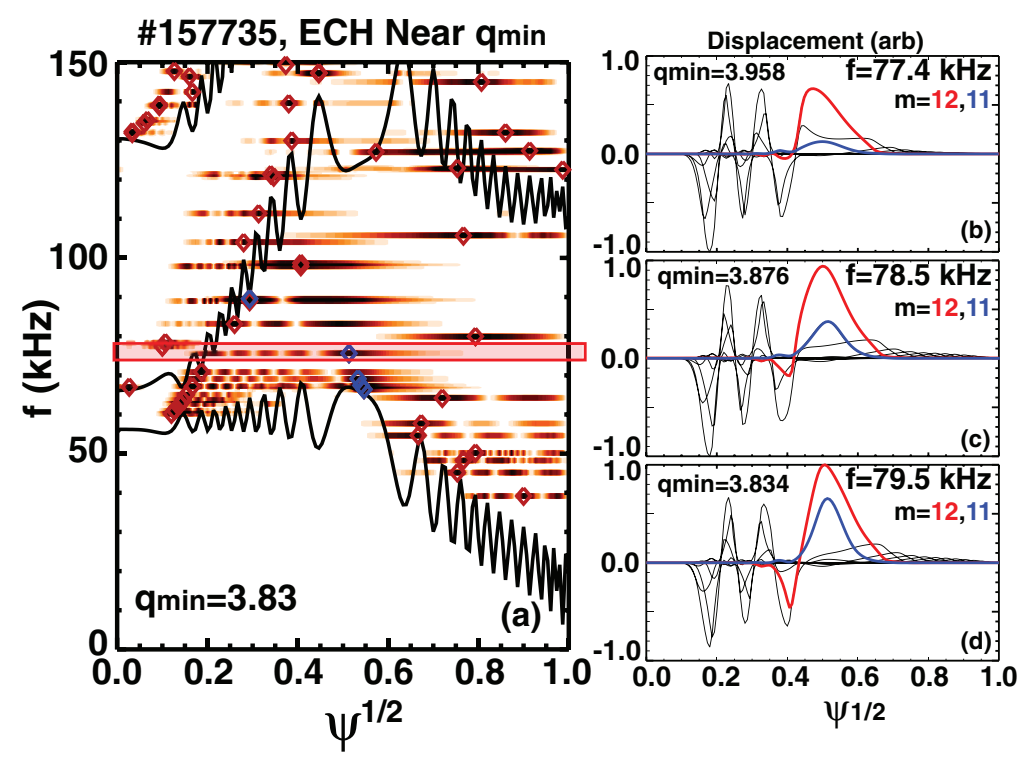

Figure 18. NOVA calculated $n=3$ continuum and eigenmodes for 157735 at $q_{\min }=3.83$. Panels $(b)-(d)$ show radial structure and poloidal decomposition of displacement for mode highlighted in red in $(a)$ at $q_{\min }=3.958,3.876$, and 3.834. The displacement color scale for panel $(a)$ is the same as shown in figure 17.

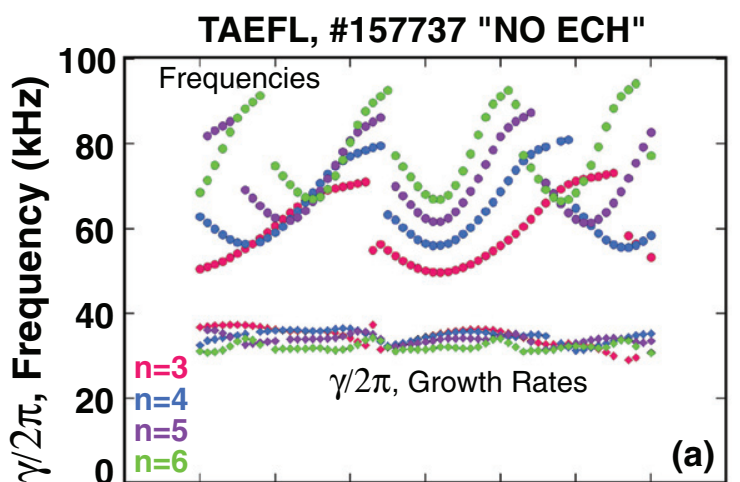

TAEFL, \#157735 "ECH Near qmin"

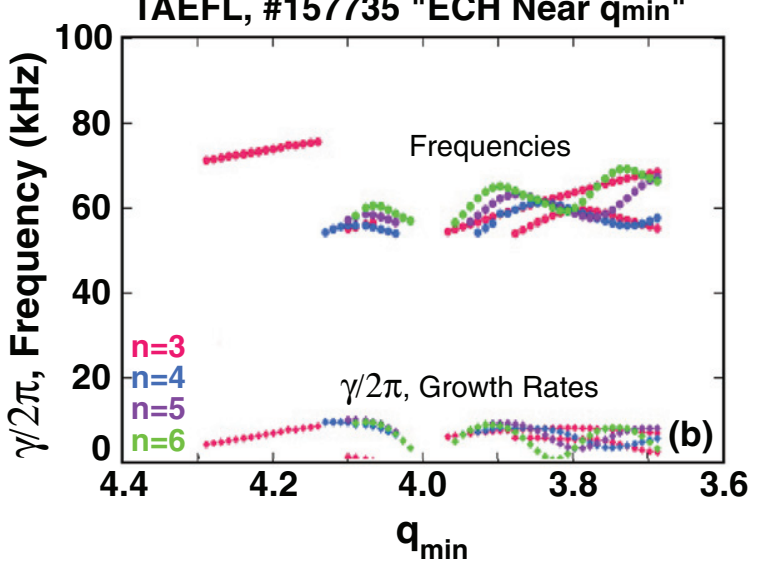

Figure 19. TAEFL calculations for DIII-D discharges $(a)$ 157737 with no $\mathrm{ECH}$ and $(b) 157735$ with $\mathrm{ECH}$ at mid-radius. Real frequencies and growth rates are shown for $n=3-6$. The corresponding experimental times are: $t \approx 370-540 \mathrm{~ms}$ and $t \approx 350$ $-480 \mathrm{~ms}$ for 157737 and 157735 respectively.

harmonic RSAEs and TAEs, very much like the spectrum of eigenmodes found by NOVA in figures 17 and 18 as well as the experimental measurements presented in figure 7 . The TAEFL calculations show not only are eigenmodes different with ECH but the calculated growth rates are also significantly lower for modes found to be unstable.

\section{Summary and conclusions}

Results have been presented from a recent DIII-D experiment and analysis focused on understanding the modification of beam driven RSAE activity by localized electron cyclotron heating. The experiments utilized a simplified oval geometry and, in addition to ECH injection location, included variations of current ramp rate, ECH injection timing, and neutral beam power. Essentially all variations carried out in this experiment were observed to change the impact of $\mathrm{ECH}$ on $\mathrm{AE}$ activity significantly. In some cases, RSAEs were observed to be more unstable with ECH near $q_{\min }$ as opposed to near the magnetic axis, in contrast to the original DIII-D experiments $[1,2]$. A database analysis of the current ramp phase in 38 discharges shows that the presence of frequency sweeping RSAEs is strongly dependent on the ratio of the RSAE minimum frequency (equation (3)) to TAE frequency (equation (1)), where the calculated RSAE minimum frequency includes an upshift from the GAM frequency (equation (4)) by an amount dependent on gradients in the local electron and ion temperatures (equation (5)). For $f_{\mathrm{RSAE}-\min } / f_{\mathrm{TAE}}>1$, no typical frequency sweeping RSAEs are observed. It is changes in this ratio through modification of electron temperature caused by $\mathrm{ECH}$ heating that is particularly effective at modifying the RSAE activity. Specifically, ECH can modify the local electron temperature at $q_{\min }$ as well as its gradient. Detailed ideal MHD calculations using the NOVA code show that by simply scaling the pressure profile of a discharge with no ECH up to that of one with ECH, typical RSAEs are no longer found. By varying both independently, NOVA simulations find that both modification of plasma pressure and pressure gradient at $q_{\min }$ play an important role in modifying the RSAE activity with 
changes in the gradient being particularly effective. Analysis of the ECH injection near $q_{\min }$ case where no frequency sweeping RSAEs are observed shows the typical RSAE is no longer an eigenmode of the system. What remains is an eigenmode with poloidal harmonic content reminiscent of the standard RSAE, but absent of the typical frequency sweeping behavior. The remaining eigenmode is also often strongly coupled to gap TAEs. Analysis with the non-perturbative gyro fluid code TAEFL confirms this change in RSAE activity and also shows a large drop in the resultant mode growth rates. While these results are very encouraging and successfully identify some of the major factors determining the impact of ECH on RSAE activity, not all of the relevant physics is taken into account and there are open avenues for research. For example, future work will focus on how the RSAE as well as continuum is modified by details of the fast ion population (which can often account for a significant fraction of the total pressure in these plasmas) and the role these effects play in determining the impact ECH has on the modes.

Localized ECH has been shown to be an effective tool for modifying Alfvén eigenmode activity in DIII-D as well as several devices worldwide [3-6]. RSAEs, being strongly influenced by finite pressure effects, are particularly sensitive to $\mathrm{ECH}$. By changing the local electron temperature and gradient at $q_{\text {min }}$, the character of the modes can be changed drastically however, it should not be assumed that application of ECH at the mode location automatically implies the stabilization/suppression of RSAEs or TAEs. In the current ramp experiments presented here, ECH also modifies the safety factor profile due to changes in the current penetration, altering both the $q_{\text {min }}$ location, mode width, and damping. Electron cyclotron current drive (ECCD) can also cause changes in the current profile and, in recent DIII-D steady state experiments, ECCD was observed to significantly reduce AE activity and improve performance [68]. Future work will focus on understanding this case and how the combination of electron cyclotron heating and current drive could be better utilized to control AE activity in a predictable way.

\section{Acknowledgments}

The work presented was carried out with support from the ITPA Energetic Particles Topical Group and contributes to the ITPA joint experiment EP-7 ('The Impact of Localized ECH on Alfvén Eigenmode Activity'). This material is based upon work supported in part by the U.S. Department of Energy, Office of Science, Office of Fusion Energy Sciences, using the DIII-D National Fusion Facility, a DOE Office of Science user facility, under Awards DE-FC02-04ER54698 ${ }^{1}$, SC-G903402², DE-AC02-09CH11466 ${ }^{7}$, DE-AC05-00OR22725 ${ }^{4}$. The work of S.E. Sharapov was funded by RCUK Energy Programme [grant EP/I501045].

\section{References}

[1] Van Zeeland M.A. 2008 Plasma Phys. Control. Fusion $\mathbf{5 0} 035009$

[2] Van Zeeland M.A. et al 2009 Nucl. Fusion 49065003
[3] Nagasaki K. et al 2012 Proc. 24th Int. Conf. on Fusion Energy (San Diego, USA, 8-13 October 2012) EX/P8-10 wwwnaweb.iaea.org/napc/physics/FEC/FEC2012/index.htm

[4] Nagaoka K. et al 2013 Nucl. Fusion 53072004

[5] Toi K. 2013 NIFS, private conversation

[6] Garcia-Munoz M. et al 2015 14th IAEA Technical Meeting (TM) on Energetic Particles in Magnetic Confinement Systems (Vienna, Austria, 1-4 Septermber, 2015) wwwnaweb.iaea.org/napc/physics/meetings/TM49508.html

[7] Kusama Y. et al 1998 Nucl. Fusion 381215

[8] Cheng C.Z., Chen L. and Chance M.S. 1985 Ann. Phys., NY 16121

[9] Cheng C.Z. and Chance M.S. 1986 Phys. Fluids 293695

[10] Fu G.Y. and Van Dam J.W. 1989 Phys. Fluids B 11949

[11] Basss E.M. and Waltz R.E. 2010 Phys. Plasmas 17112319

[12] Gorelenkov N.N. and Sharapov S.E. 1992 Phys. Scr. 45163

[13] Candy J. 1996 Plasma Phys. Control. Fusion 38795

[14] Berk H.L., Van Dam J.W., Guo Z. and Lindberg D.M. 1992 Phys. Fluids B 41806

[15] Saigusa M. et al 1997 Nucl. Fusion 371559

[16] Podesta M., Bell R.E., Fredrickson E.D., Gorelenkov N.N., LeBlanc B.P., Heidbrink W.W., Crocker N.A., Kubota S. and Yuh H. 2010 Phys. Plasmas 17122501

[17] Chu M.S., Greene J.M., Lao L.L., Turnbull A.D. and Chance M.S. 1992 Phs. Fluids B 43713

[18] Winsor N., Johnson J.L. and Dawson J.M. 1968 Phys. Fluids 112448

[19] Sharapov S.E. et al 2001 Phys. Lett. A 289127

[20] Berk H.L., Borba D.N., Breizman B.N., Pinches S.D. and Sharapov S.E. 2001 Phys. Rev. Lett. 871085002

[21] Sharapov S.E. et al 2002 Phys. Plasmas 92027

[22] Breizman B.N. et al 2003 Phys. Plasmas 103649

[23] Kramer G.J., Gorelenkov N.N., Nazikian R.M. and Cheng C.Z. 2004 Plasma Phys. Control. Fusion 46 L23

[24] Breizman B.N., Pekker M.S., Sharapov S.E. and JET EFDA contributors 2005 Phys. Plasmas 12112506

[25] Fu G.Y. and Berk H.L. 2006 Phys. Plasmas 13052502

[26] Gorelenkov N.N., Kramer G.J. and Nazikian R.N. 2006 Plasma Phys. Control. Fusion 481255

[27] Breizman B.N. 2006 Theory of Fusion Plasmas: Joint Varenna-Lausanne Int. Workshop (Varenna, Italy, 28 August-1 September 2006) CP871 http://varennalausanne.epfl.ch/Varenna2006/

[28] Kramer G.J. et al and JET EFDA Contributors 2006 Phys. Plasmas 13056104

[29] Edlund E.M., Porkolab M., Kramer G.J., Lin L., Lin Y. and Wukitch S.J. 2009 Phys. Plasmas 16056106

[30] Yu L., Zhang X. and Sheng Z.-M. 2013 Phys. Plasmas 20082509

[31] Fesenyuk O.P., Kolesnichenko Ya.I. and Yakovenko Yu.V. 2013 Phys. Plasmas 20122503

[32] Fredrickson E.D., Crocker N.A., Gorelenkov N.N., Heidbrink W.W., Kubota S., Levinton F.M., Yuh H., Menard J.E. and Bell R.E. 2007 Phys. Plasmas 14102510

[33] Lao L.L., St John H.E., Stambaugh R.D., Kellman A.G. and Pfeiffer W. 1985 Nucl. Fusion 251611

[34] Austin M.E. and Lohr J. 2003 Rev. Sci. Instrum. 741457

[35] Van Zeeland M.A. et al 2011 Phys. Plasmas 18056114

[36] Van Zeeland M.A. et al 2012 Nucl. Fusion 52094023

[37] Spong D.A. et al 2012 Phys. Plasmas 19082511

[38] Deng W. 2012 Nucl. Fusion 52043006

[39] Wang Z., Lin Z., Holod I., Heidbrink W.W., Tobias B., Van Zeeland M. and Austin M.E. 2013 Phys. Rev. Lett. 111145003

[40] Bass E.M. and Waltz R.E. 2013 Phys. Plasmas 20012508

[41] Todo Y., Van Zeeland M.A., Bierwage A. and Heidbrink W.W. 2014 Nucl. Fusion 54104012

[42] Cheng C.Z. and Chance M.S. 1987 J. Comput. Phys. 71124

[43] Cheng C.Z. 1992 Phys. Rep. 2111

[44] Spong D.A. et al 1992 Phys. Fluids B 43316 
[45] Van Zeeland M.A., Kramer G.J., Austin M.E., Boivin R.L., Heidbrink W.W., Makowski M.A., McKee G.R., Nazikian R., Solomon W.M. and Wang G. 2006 Phys. Rev. Lett. 97135001

[46] Van Zeeland M.A., Austin M.E., Gorelenkov N.N., Heidbrink W.W., Kramer G.J., Makowski M.A., McKee G.R., Nazikian R., Ruskov E. and Turnbull A.D. 2007 Phys. Plasmas 14056102

[47] Sharapov S.E. et al 2004 Phys. Rev. Lett. 93165001

[48] Van Zeeland M.A. et al 2006 Nucl. Fusion 46 S880

[49] Classen I.G.J. et al and The ASDEX Upgrade Team 2011 Plasma Phys. Control. Fusion 53124018

[50] Garcia-Munoz M. et al 2011 Nucl. Fusion 51103013

[51] Zonca F., Chen L. and Santoro R.A. 1996 Plasma Phys. Control. Fusion 382011

[52] Chavdarovski I. and Zonca F. 2009 Plasma Phys. Control. Fusion 51115001

[53] Lauber Ph., Brüdgam M., Curran D., Igochine V., Sassenberg K., Günter S., Maraschek M., Garcia-Munoz M., Hicks N. and The ASDEX Upgrade Team 2009 Plasma Phys. Control. Fusion $\mathbf{5 1} 124009$

[54] Lauber P. 2015 private communication $(n=3 R S A E$ benchmark case Max-Planck-Princeton Center for Plasma Physics, Meeting (9-12 March))

[55] Gao Z., Peng L., Wang P., Dong J. and Sanuki H. 2009 Nucl. Fusion 49045014

[56] Heidbrink W.W. 2008 Phys. Plasmas 15055501
[57] White R.B., Gorelenkov N., Heidbrink W.W. and Van Zeeland M.A. 2010 Phys. Plasmas 17056107

[58] White R.B., Gorelenkov N., Heidbrink W.W. and Van Zeeland M.A. 2010 Plasma Phys. Control. Fusion 52045012

[59] Collins C.S., Heidbrink W.W., Austin M.E., Kramer G.J., Pace D.C., Petty C.C., Stagner L., Van Zeeland M.A., White R.B., Zhu Y.B. and DIII-D Team 2016 Phys. Rev. Lett. 116095001

[60] Collins C.S. 2016 private conversation (Work to be presented at 2016 IAEA FEC, Kyoto, Japan)

[61] Heidbrink W.W., Austin M.E., Spong D.A., Tobias B.J. and Van Zeeland M.A. 2013 Phys. Plasmas 20082504

[62] Kramer G.J., Saigusa M., Ozeki T., Kusama Y., Kimura H., Oikawa T., Tobita K., Fu G.Y. and Cheng C.Z. 1998 Phys. Rev. Lett. 802594

[63] Fu G.Y., Nazikian R., Budny R. and Chang Z. 1998 Phys. Plasmas 54284

[64] Crocker N.A. et al 2008 Phys. Plasmas 15102502

[65] Turnbull A.D., Strait E.J., Heidbrink W.W., Chu M.S., Duong H.H., Greene J.M., Lao L.L., Taylor T.S. and Thompson S.J. 1993 Phys. Fluids B 52546

[66] Heidbrink W.W., Strait E.J., Chu M.S. and Turnbull M.S. 1993 Phys. Rev. Lett. 71855

[67] Classen I.G.J. et al 2010 Rev. Sci. Instrum. 81 10D929-1

[68] Petty C.C. et al 2015 21st Topical Conf. on Radiofrequency Power in Plasmas (Lake Arrowhead, CA, 27-29 April 2015) https://fusion.gat.com/conferences/rfppc2015/index.html 